Citation: Hirt, C., M. Yang, M. Kuhn, B. Bucha, A. Kurzmann and R. Pail (2019), SRTM2gravity: an ultrahigh resolution global model of gravimetric terrain corrections, Geophysical Research Letters 46, doi: 10.1029/2019GL082521.

\title{
SRTM2gravity: an ultrahigh resolution global model of gravimetric terrain corrections
}

\author{
Christian Hirt ${ }^{1,2}$, Meng Yang ${ }^{1}$, Michael Kuhn³ ${ }^{3}$ Blažej Bucha ${ }^{4}$, Andre Kurzmann ${ }^{5}$, Roland Pail ${ }^{1}$ \\ ${ }^{1}$ Institute for Astronomical and Physical Geodesy (IAPG), Technical University Munich, Arcisstr 21, 80333 Munich, Germany \\ ${ }^{2}$ Institute for Advanced Study (IAS), Technical University Munich, Lichtenbergstraße 2a 85748 Garching, Germany \\ ${ }^{3}$ School of Earth and Planetary Sciences \&Western Australian Geodesy Group, Curtin University, Perth, GPO Box U1987, Perth, WA 6845, \\ Western Australia
}

${ }^{4}$ Department of Theoretical Geodesy, Slovak University of Technology in Bratislava, Radlinského 11, 81005 Bratislava, Slovak Republic

${ }^{5}$ Leibniz-Rechenzentrum (LRZ) der Bayerischen Akademie der Wissenschaften, Boltzmannstraße 1, 85748 Garching, Germany

Corresponding Author: Christian Hirt, Email: c.hirt@tum.de

\section{Key Points}

- Global 3" SRTM topography has been accurately converted to implied topographic gravity effects at $\sim 28$ billion locations over most land areas

- $90 \mathrm{~m}$ detailed gravimetric terrain correction grid reflecting the gravitational attraction of Earth's topographic masses publicly available

- New model directly applicable to reduce gravimetric surveys to Bouguer gravity over land areas between $-60^{\circ}$ and $85^{\circ}$ geographical latitude

\begin{abstract}
We present a new global model of spherical gravimetric terrain corrections which take into account the gravitational attraction of Earth's global topographic masses at 3" ( 90 m) spatial resolution. The conversion of Shuttle Radar Topography Mission (SRTM)-based digital elevation data to implied gravity effects relies on the global evaluation of Newton's law of gravitation, which represents a computational challenge for 3 " global topography data. We tackled this task by combining spatial and spectral gravity forward modelling techniques at the $0.2 \mathrm{mGal}$ accuracy level and used advanced computational resources in parallel to complete the 1 million CPU-hour-long computation within 2 months. Key outcome is a 3" map of topographic gravity effects reflecting the total gravitational attraction of Earth's global topography at 28 billion computation points. The data, freely available for use in science, teaching and industry, is immediately applicable as new in-situ terrain correction to reduce gravimetric surveys around the globe.
\end{abstract}




\section{Plain Language Summary}

Measurement and study of the gravitational force (the g-value) is essential for geoscientists concerned with, e.g., mineral prospection and investigation of Earth's gravitational field. Most applications require the analyst to remove the gravitational signal caused by the surrounding and remote terrain (mountains, valleys) from the g-value at the location of the measurement. This task involves tedious numerical computations when high-resolution terrain data sets, e.g., from the Shuttle Radar Topography Mission (SRTM) are used. Utilizing improved computational methods and 1 million computation hours on a supercomputer, a globally $90 \mathrm{~m}$-detailed map has been created that shows the subtle influence of the terrain on g-measurements at $\sim 28$ billion measurement sites around the globe. This first-of-its-kind map, released into the public domain, is expected to simplify the daily work of geoscientists in research and industry concerned with gravity interpretation and to clear the path for next-generation global gravity maps with extreme detail.

\section{Keywords}

Earth's gravity field, gravity, terrain correction, Bouguer anomaly, gravity forward modelling, digital elevation model, SRTM

\section{Introduction}

Gravity field observations are essential for investigating the structure of Earth's gravitational field. The shape and anomalies of the gravity field carry important clues on the mass composition and geological evolution (e.g., Blakeley, 1996; Fowley, 2005). Before a gravimetric survey can be interpreted for anomalous signals, the effect of the topographic masses on the gravity measurements must be calculated and reduced. This is also denoted topographic mass reduction (Jacoby \& Smilde 2009) or gravimetric terrain correction (Li \& Sideris, 1994; Featherstone \& Kirby, 2002). The gravity effect associated with the topographic masses is obtained through evaluation of Newton's law of gravitation. While classical terrain corrections rely on approximations such as planarization and neglect of topographic masses beyond some fixed integration radius, e.g., $167 \mathrm{~km}$ (e.g., Hammer, 1939; Nowell, 1999), contemporary approaches are often based on more accurate spherical approximation and model all of Earth's topographic masses around the globe and with increasingly higher resolution (e.g., Kuhn et al., 2009; Balmino et al., 2012). The spatial resolution of terrain correction computations is important to better resolve and detect small-scale or near-surface mass-density anomalies, e.g., in the context of geophysical exploration.

In modern terrain correction computations, detailed digital elevation models (DEM) are commonly used as representation of the topographic masses. High-resolution gravimetric terrain correction grids have been computed over local (e.g., Tsoulis, 2001; Cella, 2015) and even continental areas 
(Featherstone \& Kirby, 2002; Kuhn et al., 2009) at a resolution commensurate with the DEM (e.g., $\sim 50 \mathrm{~m}$ to $\sim 270 \mathrm{~m}$ ). Global grids of gravimetric terrain corrections have been developed too, notably in the context of UNESCO's World Gravity Map (WGM) project (Balmino et al., 2012; Bonvalot et al., 2012). However, a $\sim 2$ to $\sim 4 \mathrm{~km}$ resolution level - as in case of the WGM - is usually not sufficient to accurately reduce ground gravimetric observations that capture the gravitational attraction of the surrounding local masses too. Thus far, a global map of highly-detailed gravimetric terrain corrections that would take into account the global topography to finest detail is not available. This might be related to the significant computational challenges encountered when attempting to evaluate Newton's integral down to the DEM resolution globally (Hirt et al., 2013).

Here we present the first ultra-high resolution model of gravimetric terrain corrections that uniquely unites local detail resolution with global coverage. We have converted global $90 \mathrm{~m} \mathrm{DEM}$ data, primarily based on the Shuttle Radar Topography Mission (SRTM), to implied topographic gravity effects. The outcome, denoted SRTM2gravity, is a modern gravimetric terrain correction model that reflects the gravitational attraction of Earth's global topographic masses at any of the $~ 28$ billion computation points covering all of Earth's land areas within $-60^{\circ}$ to $85^{\circ}$ geographic latitude at $90 \mathrm{~m}$ resolution. The SRTM2gravity model contains implicitly the effect of the Bouguer shell (the linear term) and all gravity terrain effects residual to the Bouguer shell (e.g., surrounding valleys, mountains). It therefore reflects the total gravity signal generated by the global topographic masses. The SRTM2gravity model relies on improved global DEM data representing the bare ground (Sect. 2) and a validated combination of mature spectral and spatial techniques for efficient evaluation of Newton's integral (Sect. 3). We provide two products, one reflecting the total gravitational attraction of the global topography, and the other capturing the high-frequency topographic gravity signal only (Sect. 4.1). Application examples including the in-situ reduction of gravimetric surveys around the globe, and construction of extremely detailed gravity maps are given (Sect 4.2), limitations are summarized (Sect 4.3), and conclusions are drawn (Sect 5).

\section{Data}

Key input data is the 3 arc-second resolution global v1.0.1 MERIT (Multi-Error-Removed ImprovedTerrain) DEM (digital elevation model) data set by Yamazaki et al. (2017). The MERIT DEM primarily relies on SRTM (Shuttle Radar Topography Mission) version 2.1 data within $\pm 60^{\circ}$ latitude, and uses AW3D DEM data (ALOS/PRISM) North of $60^{\circ}$ latitude. For the filling of SRTM voids (unobserved areas), DEM data collected and maintained by Viewfinder Panoramas was used. Together with a constant mass-density of $2670 \mathrm{~kg} \mathrm{~m}^{-3}$, MERIT is our representation for Earth's topographic masses. In contrast to other global DEM products, radar error sources (speckle noise, stripes and biases) as well as the tree canopy signal have been reduced in MERIT (see Yamazaki et al., 2017 for details). As a 
result, MERIT elevations represent - in good approximation - the bare ground, and thus improve the representation of topographic masses, which is an important conceptional benefit for the purpose of our work. The lower bound of the MERIT topography model is the geoid (mean sea level).

In previous studies using earlier SRTM data releases (e.g., Hirt et al., 2014), the need to carefully inspect and correct the topography data for outliers prior to the forward modelling has become clear. For this study, efforts were therefore made to develop artefact screening techniques (Hirt 2018) for detection of steps, spikes, pits and other unwanted features in the topography model. Based on the terrain gradient threshold of $5 \mathrm{~m} / \mathrm{m}$ (i.e., a $5 \mathrm{~m}$ elevation change per $1 \mathrm{~m}$ horizontal distance), a total of 123 locations with elevation outliers were detected in the MERIT V1.0.1 data set and removed through interpolation (Hirt, 2018). Though the number of detected artefacts is comparatively small, we consider the DEM screening and artefact removal important, because elevation outliers may propagate into a wider region of surrounding gravity values. The cleaned 3" MERIT DEM is free of spurious artefacts and represents the detailed topographic mass model (Fig. 1a) for the gravity forward modelling.

SRTM2gravity uses the MERIT-DEM as only input data set, so does not include the topographic masses of Antarctica, nor the ice-density contrast of Greenland. These simplifications produce very long-wavelength signals on the order of few mGal over non-ice-covered areas (Kuhn \& Hirt, 2016). A correction is possible, e.g., using forward models representing the ice-density effect of Greenland (Tenzer et al., 2010) and topography/ice masses of Antarctica (Rexer et al., 2016). The MERIT-DEM represents the surface of water bodies (oceans or lakes) and the surface of ice masses where present. Note that no attempt was made to model other mass-bodies or mass-density anomalies such as ocean or lake water or sediments. Users having detailed models of such mass bodies at hand can forward-model and refine the terrain corrections from the SRTM2gravity model.

\section{Methods and computations}

The principal difficulty for global yet high-resolution terrain correction modelling is the computational effort associated with evaluation of Newton's integral. The effort increases linearly with (i) the number of computation points, and (ii) the number of mass-elements the topography model is divided into. At $90 \mathrm{~m}$ spatial resolution, the MERIT-DEM contains 28 billion elevation points across all land areas within $-60^{\circ}$ and $85^{\circ}$ latitude, requiring - in principle $\sim\left(28 \times 10^{9}\right)^{2}$ evaluations of single mass elements. Using cascading grid resolutions, starting from 3 " in the vicinity of the computation point to much coarser resolutions for remote topographic masses, is permitted to accelerate the computations (e.g. Forsberg, 1984), while keeping approximation errors small (e.g., Hirt \& Kuhn, 2014). Nonetheless, with this optimized configuration (cf. Electronic Supplementary Materials ESM) the conversion of the entire MERIT-DEM to gravity 
effects would still require $\sim 15$ million CPU hours (CPUh) to obtain $\mu$ Gal level precision. Using much coarser grid resolutions would further reduce the computation time, however, at the expense of increasing approximation errors.

An alternative highly-efficient strategy was developed, tested and applied for the SRTM2gravity challenge, considerably reducing the overall computation time to the level of $\sim 1$ million CPUh. Our computational approach (cf. ESM) combines spectral-domain and spatial-domain techniques for efficient evaluation of Newton's integral. Key element of the combination technique is the use of a spherical harmonic (SH) reference topographic surface, which is here expanded up to degree 2,160. Under some approximations (cf. Rexer et al., 2018; Hirt et al., 2019, also see ESM Sect. S1 and S3), it facilitates the separate modelling of long-wavelength (here more than $10 \mathrm{~km}$ ) and short-wavelength (less than $10 \mathrm{~km}$ ) topographic gravity signals, based on the following procedure:

1. The 3" MERIT topographic surface was accurately expanded into a set of SH coefficients to degree 2,160 . We performed an ultra-high degree SH analysis up to degree 43,200 to mitigate downsampling errors on the estimated coefficients (Hirt et al., 2019). The reference surface is rigorously self-consistent with the 3 "MERIT topographic surface.

2. For modelling the long-wavelength gravity signal implied by the degree-2,160 SH topography, spectral-domain techniques as described in, e.g., Chao \& Rubincam (1989), Hirt \& Kuhn (2014) were used. These expand the topographic potential into integer powers of the topography, and gravity effects are subsequently obtained via accurate $\mathrm{SH}$ synthesis of the topographic potential coefficients at the 3" MERIT topographic surface (e.g., Hirt, 2012; Bucha \& Janak, 2014).

3. The MERIT-implied gravity signal residual to the degree-2,160 reference topography was computed in the spatial domain via local numerical integration. A residual terrain model (RTM; Forsberg, 1984) was formed as difference between the 3" MERIT and the reference topography, and converted to high-frequency gravity effects by evaluating Newton's integral locally. For this task, the RTM was subdivided into primitive mass elements - polyhedra, prisms and tesseroids (e.g., Heck \& Seitz, 2007; Tsoulis, 2012) - and the gravitational effect of each mass element was calculated and added up. Where 3" MERIT elevations were smaller than the reference topographic elevations, a harmonic correction was applied following the approach by Forsberg \& Tscherning (1981).

The total gravitational effect of the 3" MERIT topographic mass model is the sum of results from step 2) and step 3). To improve the spectral separation between both components, and to be able to reach sub-mGal modelling accuracy, also very high-frequency signals generated by the degree-2,160 
topography at scales of $\sim 10 \mathrm{~km}$ down to $\sim 2 \mathrm{~km}$ were explicitly modelled and considered (Hirt et al., 2016; Rexer et al., 2018), also see ESM (Sect. S1.3).

As the main computational benefit of the adopted methodology, the RTM numerical integration could be restricted to a comparatively small $\sim 40 \mathrm{~km}$ radius around the computation point without compromising the modelling quality (beyond the chosen integration radius, gravitational effects cancel out to a large extent because of the oscillating nature of the residual terrain). This has allowed a very significant reduction of the number of mass elements and the total computation time compared to more tedious global evaluations (radius of 20,000 km) of Newton's integral over the 3" MERIT DEM. For the production of the SRTM2gravity model, the SuperMUC Phase 2 advanced computational resources of the Leibniz Rechenzentrum (LRZ) of the Bavarian Acadamy of Sciences and humanities could be used. This part of the LRZ supercomputer comprises 86,016 CPUs (type Haswell Xeon Processor E5-2697). At 28 billion computation points,

- the spectral gravity forward modelling including synthesis of gravity effects at the 3D topographic surface required $\sim 45,000 \mathrm{CPU}$-hours $(4 \%)$, and

- the residual terrain modelling including the numerical integration within $40 \mathrm{~km}$ caps around each point required $\sim 1,100,000$ CPU-hours ( $96 \%$ of total CPU time),

showing that the majority of CPU-hours is consumed by the numerical integration, while the effort associated with spectral modelling of the long-wavelength reference signal tends to be negligible. Using up to 4,000 CPUs in parallel, the computations - which would have otherwise taken 100 years on a single-core desktop computer - could be completed in 8 weeks (gross time). Compared to a truly global evaluation of Newton's law of gravitation at 3" resolution, our spectral-spatial combination method has significantly reduced computation times. This comes at the expense of increased approximation errors over deeply carved mountain valleys, related to the approximate character of the harmonic correction in the RTM (cf. Sect 4.3 and ESM). Notwithstanding, a high precision of 0.2 mGal for the SRTM2gravity conversion could be reached globally (cf. Sect. 4.2). As an important conceptual benefit of the chosen method, a spectral separation between long- and short-wavelength topographic gravity signals is directly given, enhancing the applicability of the SRTM2gravity model, e.g., for augmentation of global gravitational models, such as EIGEN-6C4 (Förste et al., 2015) or EGM2008 (Pavlis et al. 2012). 


\section{Results}

\subsection{SRTM2gravity products and applications}

The first and primary outcome of the SRTM2gravity project is a 3" resolution global grid of gravimetric terrain corrections (Fig. 1b) which reflect the gravity signal produced Earth's global topography (Fig. 1a), excluding the land masses of Antarctica and the Greenland ice-density contrast.
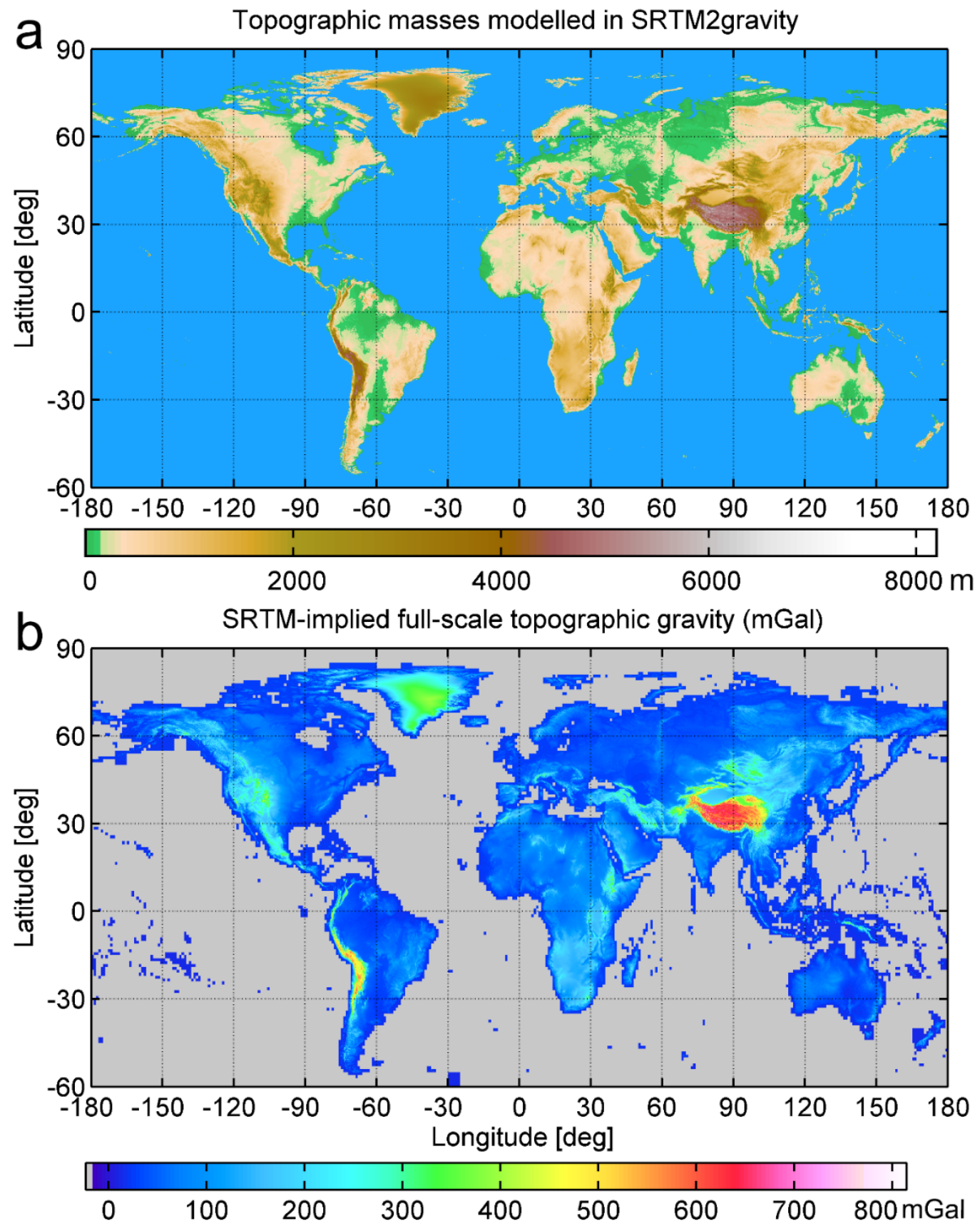

Fig. 1. Top: Global topographic masses (elevations in $\mathrm{m}$ ) modelled in SRTM2gravity, Bottom: topographyimplied full-scale gravity signal (in mGal) and model availability. Data shown at 1 arc-min resolution in both panels. 
It can be used as a modern kind of terrain correction to reduce the topographic gravitational effect in gravity measurements taken anywhere on Earth's land areas with the exception of Antarctica. The SRTM2gravity gravimetric terrain corrections have been calculated at $27,938,880,000$ points. These extend over $19,4021^{\circ} \times 1^{\circ}$ tiles covering Earth's land areas between $-60^{\circ}$ and $+85^{\circ}$ latitude including coastal zones (Fig. 1a). Over our data area, the gravimetric terrain corrections reach a total mean value of $~ 86.5 \mathrm{mGal}$. The variation range is from - $48.5 \mathrm{mGal}$ (Torres del Paine, Chile) to $\sim 825.3$ mGal (Mount Everest summit), cf. Fig. 1b.

It is important to note that our gravimetric terrain corrections shown in Fig. $1 \mathrm{~b}$ contain both the linear effect of the topography on gravity (also known as Bouguer shell) as well as the non-linear effect (classically denoted as terrain correction in textbooks); so are identical with the (full) gravitational signal generated by Earth's global topography (Fig. 1a).The SRTM2gravity gravimetric terrain corrections can thus be directly subtracted from observed gravity disturbances, without the need to separately model Bouguer plate or shell effects, see Fig. 2 for examples.
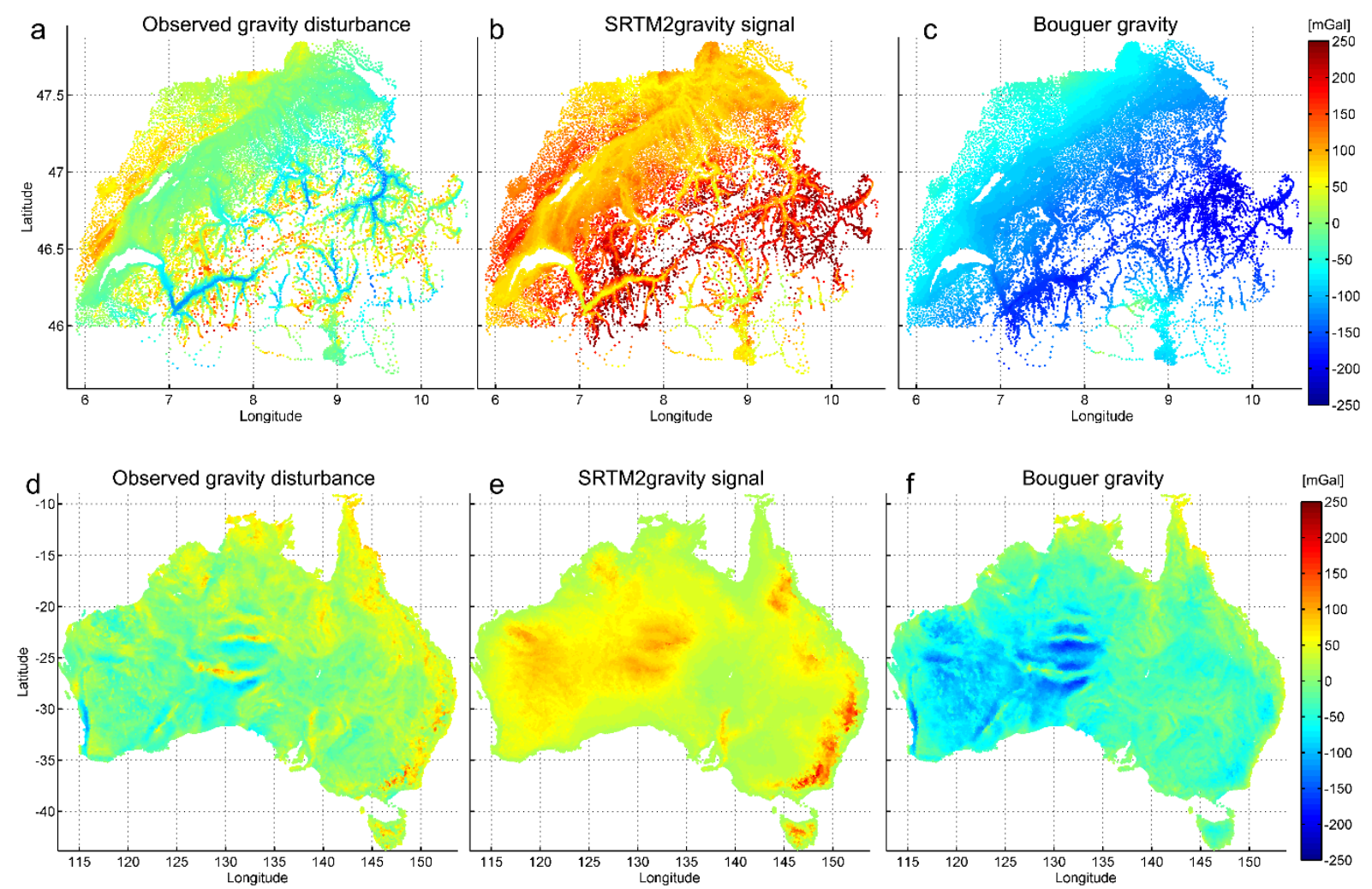

Fig. 2. Top row: Observed gravity, topographic gravity and (complete) Bouguer gravity anomalies over Switzerland; bottom row: the same but over Australia. The observations shown in panels a and d are g-values minus normal gravity, so technically gravity disturbances. The topographic gravity (panels $b, e)$ has been interpolated from the SRTM2gravity model and represents a complete spherical terrain correction. Bouguer gravity (panels c, f) follows as difference between observation and topographic effect. Data courtesy Swisstopo, and Geoscience Australia. All units in mGal.

As second outcome, we provide a 3" resolution global grid of residual gravity effects which represents high-frequency topographic gravity signals at scales from $\sim 10 \mathrm{~km}$ down to $\sim 90 \mathrm{~m}$. This 
model component is the result of the RTM numerical integration procedure (Sect. 3 ) and also takes into account very short-scale signals generated by the degree-2,160 topography to improve the band-limitation of the residual gravity signals (cf. Rexer et al. 2018). Typical applications for the residual gravity effects are a) use in the context of remove-compute-restore geoid computations to smooth gravity anomalies prior to interpolation, and b) spectral enhancement of global geopotential models (e.g., EIGEN-6C4 or EGM2008) beyond the nominal 10 km model resolution (e.g., Hirt et al., 2013). The global RMS (root-mean-square) signal strength of the residual gravity effects is $\sim 10.7$ mGal and maximum signal amplitudes often exceed $\sim 100$ to 200 mGal over rugged terrain, e.g., the Himalaya Mountains. These values corroborate the significant signal amplitudes of topographyimplied gravity signals at short spatial scales.

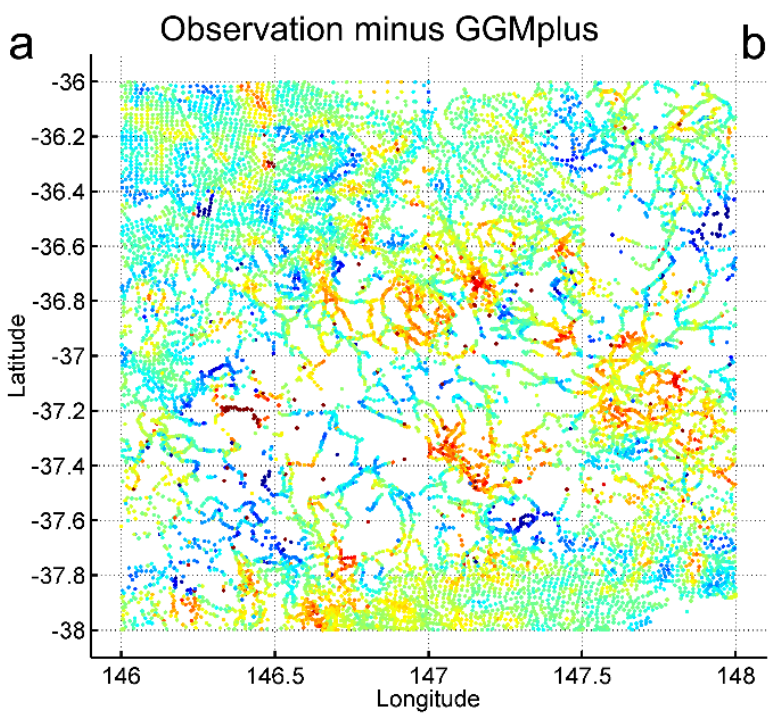

b
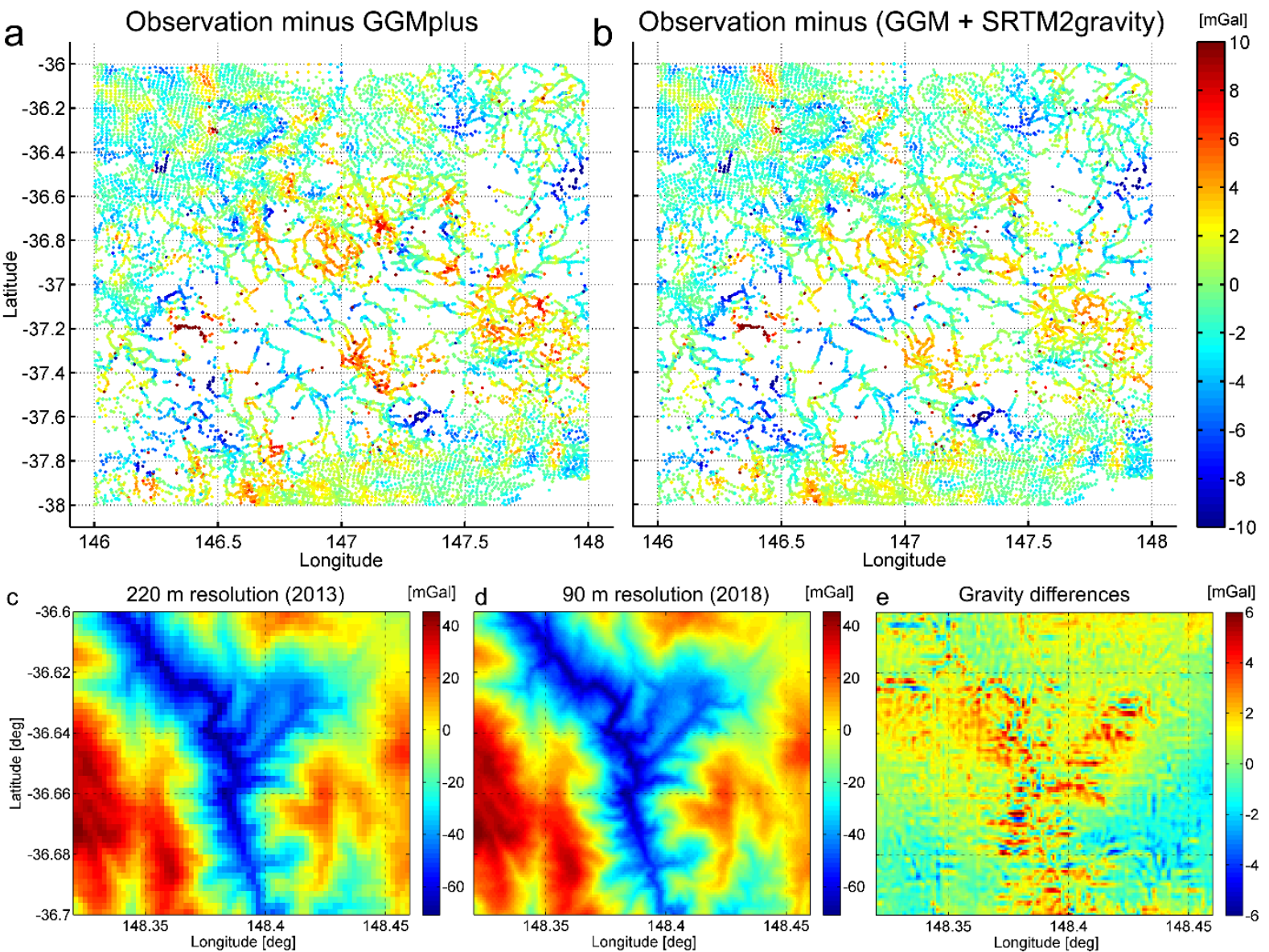

Fig. 3. Top row: Residuals between gravity observations and modelled gravity over the Australian Alps. Panel a: model = GGMplus, panel b: model = GGMplus with the short-scale component replaced by SRTM2gravity. The top row gives an example of smaller gravity residuals as result of using SRTM2gravity data. Bottom row: Comparison of the spatial detail modelled by GGMplus (Hirt et al. 2013, panel c) and SRTM2gravity (panel d) and their differences (panel e) over a $10 \times 10 \mathrm{~km}$ area of the Australian Alps. The bottom row shows the shortscale gravity model constituents and their differences (mGal) at spatial scales less than $10 \mathrm{~km}$. With the $90 \mathrm{~m}$ resolution level (centre) many short scale terrain features are better represented in the gravimetric terrain corrections. 
Fig. 2 shows application examples for SRTM2gravity products. Using the Swiss national gravity data set, Fig. $2 a$ shows free-air gravity values and Fig. $2 b$ the new gravimetric terrain corrections, which have been interpolated and subtracted from the free-air gravity to yield complete spherical Bouguer anomalies (Fig 2c). These are predominantly negative, a typical sign for isostatic compensation of the topographic masses over the European Alps. Analogously, Fig. 2d-2f shows the observed, topographic and Bouguer gravity for Australia.

The application of the SRTM2gravity residual component for the spectral enhancement of global geopotential models is exemplified in Fig. 3. Over a test area in the Australian Alps, Fig. 3a shows residuals between observed gravity and the GGMplus model (see Hirt et al., 2013), while in Fig. 3b the short-scale signals of GGMplus have been replaced with SRTM2gravity (see Table S5 from ESM for the statistics). The comparison often shows smaller residuals when SRTM2gravity is used, which is attributed here to the better spatial resolution of the forward modelling, to a reduction of canopyrelated non-gravity signals in the SRTM2gravity product, as well as to the improved spectral consistency of residual terrain modelling. Fig. 3c-d finally compares the short-scale component of GGMplus with SRTM2gravity, exemplifying the gain in resolution (from $220 \mathrm{~m}$ to $90 \mathrm{~m}$ ), and thus improved representation of short-scale gravity signals.

\subsection{Validation and accuracy}

We have independently validated the SRTM2gravity model through a) global numerical integration and b) comparisons with ground-truth gravity data (cf. ESM).

a) Over a total of six $2^{\circ} \times 2^{\circ}$ mountainous test areas around the globe (cf. ESM), global numerical integrations (= evaluation of Newton's integral in the spatial domain with $180^{\circ}$ numerical integration radius at 3 " resolution in the vicinity of the computation point) were performed. These calculations provide reference values for the gravimetric terrain correction - without using spectral techniques, spherical harmonic reference surfaces or RTM gravity forward modelling methods - so offer an independent check of the modelling technique the SRTM2gravity products rely on (Sect. 3). The RMS differences between SRTM2gravity values and reference values from global numerical integration were found to be smaller than $0.8 \mathrm{mGal}$ over all test areas, with the RMS values ranging from 0.1 mGal (Australian Alps), 0.15 mGal (Indonesian Islands and South American Andes), 0.5-0.6 mGal (European Alps and Canadian Rocky Mountains) to $0.75 \mathrm{mGal}$ (Himalayas) as example for Earth's most rugged areas. Maximum differences at individual field points never exceeded an amplitude of $12.5 \mathrm{mGal}$ over our test areas. For any area where topography is smoother than that of the Australian Alps (these are more than $90 \%$ of Earth's land areas), $\sim 0.1 \mathrm{mGal}$ accuracy can be reasonably expected. 
These values are a measure for the computational accuracy, in that, they show the error level that can be attributed to our SRTM2gravity conversion technique described in Sect. 3. SRTM2gravity values should be well reproducible within this error margin with any independent and accurate forward modelling technique. It is important to bear in mind that the error level of SRTM elevation data is $\sim 5 \mathrm{~m}$ (e.g., Rodriguez et al. 2006), which translates into $\sim 0.5 \mathrm{mGal}$ in the gravity domain (using a simple Bouguer plate model). In rugged terrain, individual SRTM errors can exceed 100 meters (or $\sim 10 \mathrm{mGal})$. Relative to the impact of elevation errors on the terrain corrections, the SRTM2gravity computational error level will thus play only a minor role for the quality of the terrain corrections.

b) We have utilized the GGMplus gravity maps which rely on SRTM-based forward modelling at spatial scales of $\sim 10 \mathrm{~km}$ down to $250 \mathrm{~m}$, and EGM2008 including GRACE/GOCE satellite gravity data at spatial scales down to $10 \mathrm{~km}$. Ground-truth gravity was compared with two model variants, (1) GGMplus gravity (as released by Hirt et al., 2013), and (2) EGM2008, refined with GRACE/GOCE satellite gravity (as in Hirt et al. 2013) and augmented with the $90 \mathrm{~m}$ resolution SRTM2gravity shortscale component. Over our test areas Bavaria, Switzerland, Australia and Slovakia, the ground-truth gravity data sets suggest a comparable or better agreement (mostly over mountainous regions) when the new SRTM2gravity model is used as source for short-scale gravity constituents (see Table S5 from ESM for the statistics). This outcome can be attributed to the higher spatial detail resolution (90 m instead of $220 \mathrm{~m}$ ), the use of bare-ground elevations and refined forward modelling techniques (cf. Rexer et al., 2018).

\subsection{Limitations}

SRTM2gravity is a pure topography-implied gravity field model. Due to its very nature, it does not contain any observed gravity values. At short spatial scales, our model is an approximation of the real gravity field, but not an exact description of what can be measured with gravimetric techniques.

While due attempts have been made to remove spurious artefacts from the topographic input model we cannot exclude the presence of further smaller artefacts in the topography data (e.g., steps or spikes with terrain gradients less than $5 \mathrm{~m} / \mathrm{m}$ ), and, in turn, in the forward-modelled gravity.

SRTM2gravity models the topographic gravity effect only and relies on the constant mass-density assumption. Mass-density anomalies (relative to the reference density of $2670 \mathrm{~kg} \mathrm{~m}^{-3}$ ) and the topographic masses of Antarctica have not been modelled. Examples of unmodelled density anomalies include, but are not limited to, the density contrasts associated with a) lake water, b) ocean water, c) ice sheets and d) sediments. Users with mass density models at hand can forward model and improve the SRTM2gravity terrain correction grids. The mentioned restrictions especially apply to Greenland and to coastlines around the world. An extension of the modelling to Antarctica 
and the inclusion of ice-sheets, ocean and lake bathymetry in detailed 3" resolution forward modelling is desirable and considered an important future task.

Over extremely narrow and deep mountain valleys (e.g., $2 \mathrm{~km}$ height difference w.r.t. surrounding summits), SRTM2gravity approximation errors will be largest. This is a consequence of the harmonic correction approach applied in the RTM gravity forward modelling. Approximation errors can reach few mGal up to $12 \mathrm{mGal}$ for km-deep narrowly-carved mountain valleys, as encountered e.g., in parts of the Himalayas, Rocky Mountains or European Alps. This effect, included in the RMS accuracy estimates in Sect. 4.2, will decrease for wider valleys and less rugged topography.

\section{Discussion and conclusions}

SRTM2gravity is the first successful attempt to transform global 3" elevation data to implied gravity effects at $\sim 28$ billion computation points covering all of Earth's land areas within $-60^{\circ}$ to $85^{\circ}$ geographic latitude, and to release the grids into the public domain for free use. SRTM2gravity is based on an efficient computational methodology (Sect. 3) that was applied in a globally consistent manner on a supercomputing facility.

The main product is a 3" global grid of gravimetric terrain corrections that is immediately applicable to reduce the topographic signal in detailed gravimetric surveys. It contains the gravity effect of the Bouguer shell and that of the terrain irregularities around the globe in a single product. As such, complete Bouguer gravity anomalies as a modern kind of Bouguer gravity are obtained (e.g., Kuhn et al. 2009). Different to classical planar approaches that often use $\sim 167 \mathrm{~km}$ integration zones (e.g., Leaman, 1998; Nowell, 1999; Fowler, 2005; Torge \& Müller, 2012; Pasteka et al., 2017), our new gravimetric terrain corrections take into account the gravitational attraction of the topographic masses around the globe in spherical approximation. As a result, the topography-implied gravity effect is modelled much more completely and realistically.

The SRTM2gravity project can be thought of a logical continuation of the Kuhn et al. (2009) study that presented gravimetric terrain corrections over Australia at $\sim 270 \mathrm{~m}$ resolution based on global numerical integration. Different to Kuhn et al. (2009), our model covers all land areas (apart from Antarctica), while now achieving $\sim 90 \mathrm{~m}$ point density, being a standard resolution for contemporary global DEM data sets. Compared to Balmino et al. (2012) who have globally modelled gravimetric terrain corrections with spectral techniques at $\sim 2 \mathrm{~km}$ resolution, our new $90 \mathrm{~m}$ SRTM2gravity grids provide a more than 20 -fold improvement in detail resolution, allowing to capture gravity signals induced by local topography too. Given the sensitivity of gravity measurements for near topographic masses, the 90 m SRTM2gravity resolution may be crucial to improve the spectral consistency with measured ground gravity. On the other hand, in favour of Balmino et al. (2012) is that their maps are 
not restricted solely to land areas and a single constant mass density, as done in this study, but take into account also density contrasts associated with bathymetry, lakes and ice caps.

SRTM2gravity is related to the GGMplus gravity maps (Hirt et al., 2013). Similarly to GGMplus, shortscale gravity signals have been modelled at scales less than $~ 10 \mathrm{~km}$ using DEM data and numerical integration techniques. SRTM2gravity, however, offers higher detail resolution (3" instead of 7.2" as in case of GGMplus), and uses improved data and modelling methods. Data improvements include a) reduced radar errors and removal of canopy signals in the DEM (Yamazaki et al., 2017), b) artefact screening and removal (Hirt, 2018) and c) coverage of high Northern latitudes (Yamazaki et al., 2017), while methodological improvements concern a more rigorous short-scale forward modelling (Hirt et al., 2019) and improved spectral consistency (Rexer et al., 2018). GGMplus primarily contained measured gravity data (via EGM08, GOCE and GRACE) at scales larger than $10 \mathrm{~km}$, whereas the SRTM2gravity model depends on topographic data at all spatial scales. As such, GGMplus was never a model of (complete) gravimetric terrain corrections, as it is now the case with SRTM2gravity.

SRTM2gravity represents a new milestone for ultra-high resolution global gravity modelling combining global scope with local detail. In the context of the new 30 m NASA-DEM, an increase in spatial resolution to the 1 " level for future gravity products is foreseeable. This will further reduce very short-scale signal omission errors in future terrain correction products. On the other hand, modelling of gravity signals associated with ice and water masses at highest possible resolution is considered important future work; issues such as outliers and inconsistencies between land topography and ocean bathymetry in coastal zones (Hirt et al., 2014) will need to be carefully addressed in such future endeavours.

\section{Acknowledgement}

This project received support by the German National Research Foundation (DFG) via grant Hi1760/01. The authors gratefully acknowledge computing time on the SuperMUC supercomputing facility by the Leibniz Supercomputing Centre (www.Irz.de) for the production of the SRTM2gravity model and the Pawsey Supercomputing Centre (www.pawsey.org.au) for the model validation. MK acknowledges Curtin University for the opportunity to contribute to this project as part of an academic study program. Providers of ground-truth data (Bayerisches Landesamt für Digitalisierung, Breitband und Vermessung, Swisstopo, Geoscience Australia) are kindly acknowledged.

\section{Data statement}

The SRTM2gravity products are freely available via ddfe.curtin.edu.au/models/SRTM2gravity2018 and further information are found at the project website http://www.bgu.tum.de/en/iapg/forschung/schwerefeld/S2g/ 


\section{References}

Balmino, G., N. Vales, S. Bonvalot and A. Briais (2012), Spherical harmonic modelling to ultra-high degree of Bouguer and isostatic anomalies, J. Geod., 86(7), 499-520, doi: 10.1007/s00190-011-0533-4.

Blakeley, R.J. (1996), Potential Theory in Gravity and Magnetic Applications, Cambridge: Cambridge University Press.

Bonvalot, S., Balmino, G., Briais, A., M. Kuhn, Peyrefitte, A., Vales, Biancale, R., Gabalda, G., Moreaux, G., Reinquin, F. Sarrailh, M. World Gravity Map, 1:50000000 map, Eds. : BGI-CGMW-CNES-IRD, Paris, 2012.

Bucha, B. and J. Janák (2014), A MATLAB-based graphical user interface program for computing functionals of the geopotential up to ultra-high degrees and orders: Efficient computation at irregular surfaces, Comp. and Geosci., 66, 219-227, doi: 10.1016/j.cageo.2014.02.005

Cella, F. (2015), GTeC - A versatile MATLAB ${ }^{\circledR}$ tool for a detailed computation of the terrain correction and Bouguer gravity anomalies, Comp. and Geosci., 84, 72-85, doi:10.1016/j.cageo.2015.07.015.

Chao, B.F. and D.P. Rubincam (1989), The gravitational field of Phobos. Geophysical Research Letters, 16(8), 859-862.

Featherstone W. E., and J. F. Kirby (2002), New high-resolution grid of gravimetric terrain corrections over Australia, Australian Journal of Earth Sciences, 49, 733-734.

Forsberg R. and C.C. Tscherning (1981), The use of height data in gravity field approximation by collocation, J. Geophys. Res., 86, 7843-7854.

Forsberg R. (1984), A study of terrain reductions, density anomalies and geophysical inversion methods in gravity field modelling. Report 355, Department of Geodetic Science and Surveying, Ohio State University, Columbus.

Förste C, Bruinsma SL., Abrikosov O et al. (2015), EIGEN-6C4 The latest combined global gravity field model including GOCE data up to degree and order 2190 of GFZ Potsdam and GRGS Toulouse. doi:10.5880/icgem.2015.1.

Fowler, C.R.M. (2005), The Solid Earth - an introduction to global geophysics, 2nd edition, Cambridge: Cambridge University Press.

Hammer, S. (1939), Terrain corrections for gravimeter stations, Geophysics, 4, 184-194.

Heck, B., and K. Seitz (2007), A comparison of the tesseroid, prism and point-mass approaches for mass reductions in gravity field modelling. J Geod., 81(2), 121-136, doi: 10.1007/s00190-006-0094-0.

Hirt, C. (2012), Efficient and accurate high-degree spherical harmonic synthesis of gravity field functionals at the Earth's surface using the gradient approach, J. Geod., 86(9), 729-744, doi:10.1007/s00190-012-0550-y.

Hirt, C., S.J. Claessens, T. Fecher, M. Kuhn, R. Pail, M. Rexer (2013), New ultra-high resolution picture of Earth's gravity field, Geophysical Research Letters, Vol 40, doi: 10.1002/grl.50838.

Hirt, C., and M. Kuhn (2014), Band-limited topographic mass distribution generates full-spectrum gravity field: Gravity forward modeling in the spectral and spatial domains revisited, J. Geophys. Res., 119(4), 3646-3661, doi: 10.1002/2013JB010900.

Hirt C., M. Kuhn, S.J. Claessens, R. Pail, K. Seitz, T. Gruber (2014), Study of the Earth's short-scale gravity field using the ERTM2160 gravity model, Computers \& Geosciences, 73, 71-80. doi: 10.1016/j.cageo.2014.09.00.

Hirt, C., E. Reußner, M. Rexer, and M. Kuhn (2016), Topographic gravity modelling for global Bouguer maps to degree 2,160: Validation of spectral and spatial domain forward modelling techniques at the 10 microgal level, J. Geophys. Res., 121(9), 6846-6862, doi: 10.1002/2016JB013249.

Hirt, C. (2018), Artefact detection in global digital elevation models (DEMs): The Maximum Slope Approach and its application for complete screening of the SRTM v4.1 and MERIT DEMs, Remote Sensing of Environment, 207, 27-41, doi:10.1016/j.rse.2017.12.037.

Hirt, C., B. Bucha, M. Yang, and M. Kuhn (2019), A numerical study of residual terrain modelling (RTM) techniques and the harmonic correction using ultra-high degree spectral gravity modelling, J. Geod. accepted for publication. 
Jacoby, W., and P.L. Smilde (2009), Gravity interpretation: Fundamentals and application of gravity inversion and geological interpretation, Springer, Berlin, Heidelberg.

Kuhn, M., W.E. Featherstone, and J.F. Kirby (2009), Complete spherical Bouguer gravity anomalies over Australia, Australian J. Earth Sci., 56, 213-223.

Kuhn, M. and C. Hirt (2016), Topographic gravitational potential up to second-order derivatives: an examination of approximation errors caused by rock-equivalent topography (RET), Journal of Geodesy, 90(9), 883-902, doi:10.1007/s00190-016-0917-6.

Leaman, D.E. (1998), The gravity terrain correction - practical considerations, Exploration Geophysics, 29, 476-471.

Li Y. C., and M. G. Sideris (1994), Improved gravimetric terrain corrections, Geophysical Journal International, 119, 740-752. Nowell, D.A.G. (1999), Gravity terrain corrections - an overview, Journal of Applied Geophysics, 42, 117-134.

Pasteka, R., J. Mikuska and B. Meurers (2017), Understanding the Bouguer Anomaly - A Gravimetry Puzzle, 1st Edition, Elsevier.

Pavlis NK, Holmes SA, Kenyon SC, Factor JK (2012) The development and evaluation of the Earth Gravitational Model 2008 (EGM2008). Journal of Geophysical Research - Solid Earth, 117(B4), B04406. doi: 10.1029/2011JB008916

Rexer, M., C. Hirt, B. Bucha and S. Holmes (2018), Solution to the spectral filter problem of residual terrain modelling (RTM), Journal of Geodesy, 92(6), 675-690, doi:10.1007/s00190-017-1086-y.

Rexer, M, C. Hirt, S.J. Claessens and R. Tenzer (2016), Layer-based modelling of the Earth's gravitational potential up to $10 \mathrm{~km}$-scale in spherical harmonics in spherical and ellipsoidal approximation, Surveys in Geophysics, 37(6), 10351074, doi:10.1007/s10712-016-9382-2.

Rodriguez, E., C.S. Morris, and J.E. Belz (2006), A global assessment of the SRTM performance. Photogrammetric Engineering \& Remote Sensing, 72(3), 249-260.

Tenzer R., A. Abdalla, P. Vajda, Hamayun (2010), The spherical harmonic representation of the gravitational field quantities generated by the ice density contrast. Contrib Geophys Geod, 40(3):207-223

Torge W., and J. Müller (2012), Geodesy. 4th Edition. Berlin, New York: W. de Gruyter.

Tsoulis D. (2001), Terrain correction computations for a densely sampled DTM in the Bavarian Alps. J Geod, 75(5-6):291307. doi: $10.1007 /$ s001900100176

Tsoulis D. (2012), Analytical computation of the full gravity tensor of a homogeneous arbitrarily shaped polyhedral source using line integrals. Geophysics, 77, F1-F11.

Yamazaki, D., D. Ikeshima, R. Tawatari, T. Yamaguchi, F. O'Loughlin, J.C. Neal, C.C. Sampson, S. Kanae, P.D. Bates (2017), A high accuracy map of global terrain elevations, Geophysical Research Letters, 44(11), 5844-5853, Doi: 10.1002/2017GL072874.

Supporting Information is provided in a separate file titled "Electronic supplementary materials (ESM) to SRTM2gravity: an ultra-high resolution global model of gravimetric terrain corrections (17 pages) 


\title{
Electronic supplementary materials (ESM) to
}

\section{SRTM2gravity: an ultra-high resolution global model of gravimetric terrain corrections}

\author{
Christian Hirt ${ }^{1,2}$, Meng Yang ${ }^{1}$, Michael Kuhn ${ }^{3}$, Blažej Bucha ${ }^{4}$, Andre Kurzmann ${ }^{5}$, Roland Pail ${ }^{1}$ \\ ${ }^{1}$ Institute for Astronomical and Physical Geodesy (IAPG), Technical University Munich, Arcisstr 21, 80333 Munich, Germany \\ ${ }^{2}$ Institute for Advanced Study (IAS), Technical University Munich, Lichtenbergstraße 2a 85748 Garching, Germany \\ ${ }^{3}$ School of Earth and Planetary Sciences \&Western Australian Geodesy Group, Curtin University, Perth, GPO Box U1987, Perth, WA 6845, \\ Western Australia \\ ${ }^{4}$ Department of Theoretical Geodesy, Slovak University of Technology in Bratislava, Radlinského 11, 81005 Bratislava, Slovak Republic \\ ${ }^{5}$ Leibniz-Rechenzentrum (LRZ) der Bayerischen Akademie der Wissenschaften, Boltzmannstraße 1, 85748 Garching, Germany
}

\section{General}

SRTM2gravity is a freely-available global model of gravimetric terrain corrections at 3" spatial resolution. The gravimetric terrain corrections reflect the gravity effect of Earth's global topographic masses, as represented through the MERIT digital elevation model together with the constant massdensity $\rho=2670 \mathrm{~kg} \mathrm{~m}^{-3}$. They include both the gravity effect of a spherical Bouguer shell and that of the terrain irregularities (e.g., valleys, summits) around the globe in a single, readily-usable data set. SRTM2gravity values facilitate a simple procedure for the compilation of Bouguer gravity maps: Our pre-computed data set can be interpolated and directly subtracted from measured gravity disturbances (differences between g-values and normal gravity) to remove the topographic signal from a gravity survey. This yields Bouguer gravity anomalies, without the need to further evaluate terrain correction integrals through tedious numerical integrations.

The purpose of these electronic supplementary materials (ESM) is to provide details on the methodology used to calculate terrain corrections from the elevation data (Sect. S1). Because the fundamental aspects of gravity forward modelling techniques are very well documented in the literature (e.g., Forsberg and Tscherning 1981, Chao and Rubincam 1989, Jacoby and Smilde 2009, Balmino et al. 2012, Hirt and Kuhn 2014, Hirt et al. 2016), we attempt to provide the specific details that we consider important to understand the challenges faced and solved, and to enable users to replicate the calculations. We briefly outline how supercomputing resources facilitated the modelling (Sect. S2), present details on the validation experiments carried out to check the correctness of the methodology, and determine the SRTM2gravity approximation error level over different topographic settings (Sect. S3). Comparisons with ground-truth data sets provide additional validation (Sect. S4).

\section{S1. Details on the methodology}

This work uses gravity forward modelling (GFM) techniques to compute the gravity field implied by Earth's topographic masses. The key input quantity for GFM in general and terrain correction computations in particular is a high-resolution topographic mass model that is defined by a detailed grid of topographic heights, together with a mass-density value $\rho$. In our case, topographic heights were taken from the MERIT-DEM (Multi-Error-Removed Improved-Terrain DEM) data set by Yamazaki et al. (2017) that relies primarily on data from the Shuttle Radar Topography Mission (SRTM). The MERIT-DEM represents the upper bound and the geoid (or mean sea level) the lower bound of the topographic mass distribution which is shown in Fig. 1a of the manuscript. In our work we use a constant mass-density value $\rho=2670 \mathrm{~kg} \mathrm{~m}^{-3}$ which is a commonly used standard value for gravimetric terrain corrections in geophysics and geodesy (e.g., Fowler 2005, Jacoby and Smilde 
2009, Torge and Müller 2012). The topography model is used in spherical approximation, i.e. with the heights $H$ referring to an Earth reference sphere with some reference radius $R$.

The gravimetric terrain correction $\delta g^{H}$ is the gravity effect implied by the MERIT topographic mass model with the computation points residing at the surface of the MERIT topography (i.e., as approximation of Earth's surface where gravity measurements can be taken). The quantity $\delta g^{H}$ is technically the first negative radial derivative of the topography-implied gravitational potential. It can be obtained via global evaluation of Newton's law of gravitation that reads in spherical approximation in integral form (after Heck and Seitz 2007)

$$
\delta g^{H}(r, \varphi, \lambda)=G \rho \int_{\psi=0}^{\psi_{0}=\pi} \int_{\alpha=0}^{2 \pi} \int_{r_{Q}=R}^{R+H} \frac{r_{Q}^{2}\left(r-r_{Q} \cos \psi\right)}{l^{3}} d r_{Q} d \alpha \sin \psi d \psi
$$

Where $G$ is the universal gravitational constant, $(H, \rho)$ is the topographic mass model, and $(r, \varphi, \lambda)$ are the radius and the geographical coordinates (spherical latitude and longitude) of the computation point $P$. Variables $\left(r_{Q}, \varphi_{Q}, \lambda_{Q}\right)$ are the radius and the geographical coordinates of the integration points $Q$, and variables $(\psi, \alpha)$ are the spherical distance and azimuth between $P$ and $Q$ which are separated by the Euclidian distance $l$. Quantity $\psi_{0}$ denotes the radius of the integration cap, with $\psi_{0}=\pi$ required for global integrations. In our work, we use $R=6378,137.0 \mathrm{~m}, G=$ $6.67384 \times 10^{-11} \mathrm{~m}^{3} \mathrm{~kg}^{-1} \mathrm{~s}^{-2}$ and $\rho=2670 \mathrm{~kg} \mathrm{~m}^{-3}$.

Because of the enormous computational effort associated with evaluation of Eq. (1) at 3" spatial resolution through numerical integration (see details in Sect. 3), we split the integral into two components

$$
\delta g^{H}=\delta g^{H_{R E F}}+\delta g^{R T M}
$$

which can be evaluated much more efficiently compared to Eq. (1). In Eq. (2),

$\delta g^{H_{R E F}}$ is the gravity signal generated by the long-wavelength constituents of the topography model, computed in our work with spectral-domain GFM techniques, and

$\delta g^{R T M}$ is the gravity signal generated by the short-wavelength constituents of the topography model, obtained through the residual terrain modelling (RTM) technique that relies on numerical integration in the spatial domain.

The combination technique (Eq. 2) confers very significant savings in computation time, primarily because the tedious numerical integration over the global topography in Eq. (1) is replaced (in a reasonable approximation) by a more efficient numerical integration within local integration caps. However, apart from the approximation due to the cap integration, the separation into two constituents in Eq. (2) must be considered as approximative, given the nonlinear relation between the topographic heights and the implied gravity. Nevertheless, after introducing correction terms, we keep the errors at least below the mGal-level (RMS) as will be shown later in the validation against an independent technique that avoids this separation (Sect. S3).

\section{S1.1 Spherical harmonic reference topography}

For the separation of long- and short-wavelength constituents of the topography model, a spherical harmonic (SH) expansion of the reference topography 


$$
H^{R E F}(\varphi, \lambda)=\sum_{n=0}^{N} \sum_{m=-n}^{n} \bar{H}_{n m} \bar{Y}_{n m}(\varphi, \lambda)
$$

is introduced, where $\bar{Y}_{n m}(\varphi, \lambda)$ are the fully-normalized surface SH functions of degree $n$ and order $m, \bar{H}_{n m}$ are the fully normalized SH coefficients of degree $n$ and order $m$, degree $N$ is the maximum degree of the $\mathrm{SH}$ expansion and $H^{R E F}(\varphi, \lambda)$ are the synthesized topographic heights. The $\mathrm{SH}$ coefficients $\bar{H}_{n m}$ were obtained via global spherical harmonic analysis (SHA) of the MERIT topography using the technique and software by Rexer and Hirt (2015). In brief, the 3" MERIT heights were block-averaged to 15 " resolution (using $5 \times 5$ block means) and subsequently analysed to ultra-high spherical harmonic degree $N=43,200$, from which we use the coefficients with $n \leq$ $N=2,160$ only. It was found that ultra-high degree SHA was required to reduce the effect of aliasing (on the estimated coefficients $n \leq N=2,160$ ) well below the sub-meter-level (cf. Hirt et al. 2019).

The coefficients $\bar{H}_{n m}$ were used to define the input topographic mass model for the spectral-domain forward modelling (S1.2) and to define the SH reference topography in the RTM forward modelling (S1.3). For the latter case, reference topographic heights $H^{R E F}(\varphi, \lambda)$ were synthesized in the spatial domain with Eq. (3) and $N=2,160$.

\section{S1.2 Spectral-domain gravity forward modelling}

Spectral-domain gravity forward modelling (e.g., Rummel et al. 1988, Chao and Rubincam 1989, Balmino et al. 2012, Hirt and Kuhn 2014; Hirt et al. 2016) involves a) the expansion of the topographic potential implied by the reference topography into integer powers $p$ of $H^{R E F}$ (S1.2.1), and b) SH synthesis of gravity effects in the space domain (S1.2.2).

\section{S1.2.1 Generation of topographic potential coefficients}

In spherical approximation, the topographic potential coefficients $V_{n m}$ implied by the reference topography $H^{R E F}$ are obtained as function of the $H_{n m}^{(p)}$ coefficients (after Chao and Rubincam 1989)

$$
V_{n m}=\frac{4 \pi R^{3} \rho}{(2 n+1) M} \sum_{p=1}^{p_{\max }} \frac{\prod_{i=1}^{p}(n-i+4)}{p !(n+3)} H_{n m}^{(p)}
$$

where $V_{n m}=\left(\bar{C}_{n m}, \bar{S}_{n m}\right)$ are the potential SHCs evaluated to $k N$ with $1 \leq k \leq p_{\text {max }}$ and $k N \in \mathbb{N}$, $H_{n m}^{(p)}$ are the SH coefficients of the topographic height function $H^{(p)}:=\left(H^{R E F} / R\right)^{p}$ obtained via SHA, $R=6378,137 \mathrm{~m}$ as model reference radius and $M=5.972 \times 10^{24} \mathrm{~kg}$ is Earth's total mass.

In our work, we used $N=2,160$, powers $1 \leq p \leq p_{\max }=40$, and $k=5$. It is well known that raising $H^{R E F} / R$ to integer power $p$ gives rise to additional short-scale signals with spectral energy in band of degrees $N+1$ to $p N$ (cf. Balmino 1994, Freeden and Schneider 1998, Hirt and Kuhn 2014) which must be taken into account to completely model the gravitational field of $H^{R E F}$ up to ultrahigh degrees. In our work, we model the gravity field implied by the $N=2,160$ reference topography up to ultra-high degree $k N=10,800$ over mountainous terrain and to degree 6,840 over the rest of the globe. Fig. S1 shows the spectral power (using degree variances as a measure for degree signal strengths of the MERIT topographic potential model) as function of the SH degree $n$.

The bulk of spectral energy is contained in spectral band of degrees $0 \leq n \leq 2,160$. In the spatial domain, gravity signals in this spectral band can reach several $100 \mathrm{mGal}$ of amplitude. Topography- 
implied gravity signals in spherical harmonic band of degrees $2,160 \leq n \leq 10,800$ can reach amplitudes of $\sim 10 \mathrm{mGal}$ or more over mountainous terrain (Hirt et al. 2016), so are taken into account here to improve the quality of the SRTM2gravity model. For more details and a justification of the chosen parameters, we refer to Hirt et al. (2016).

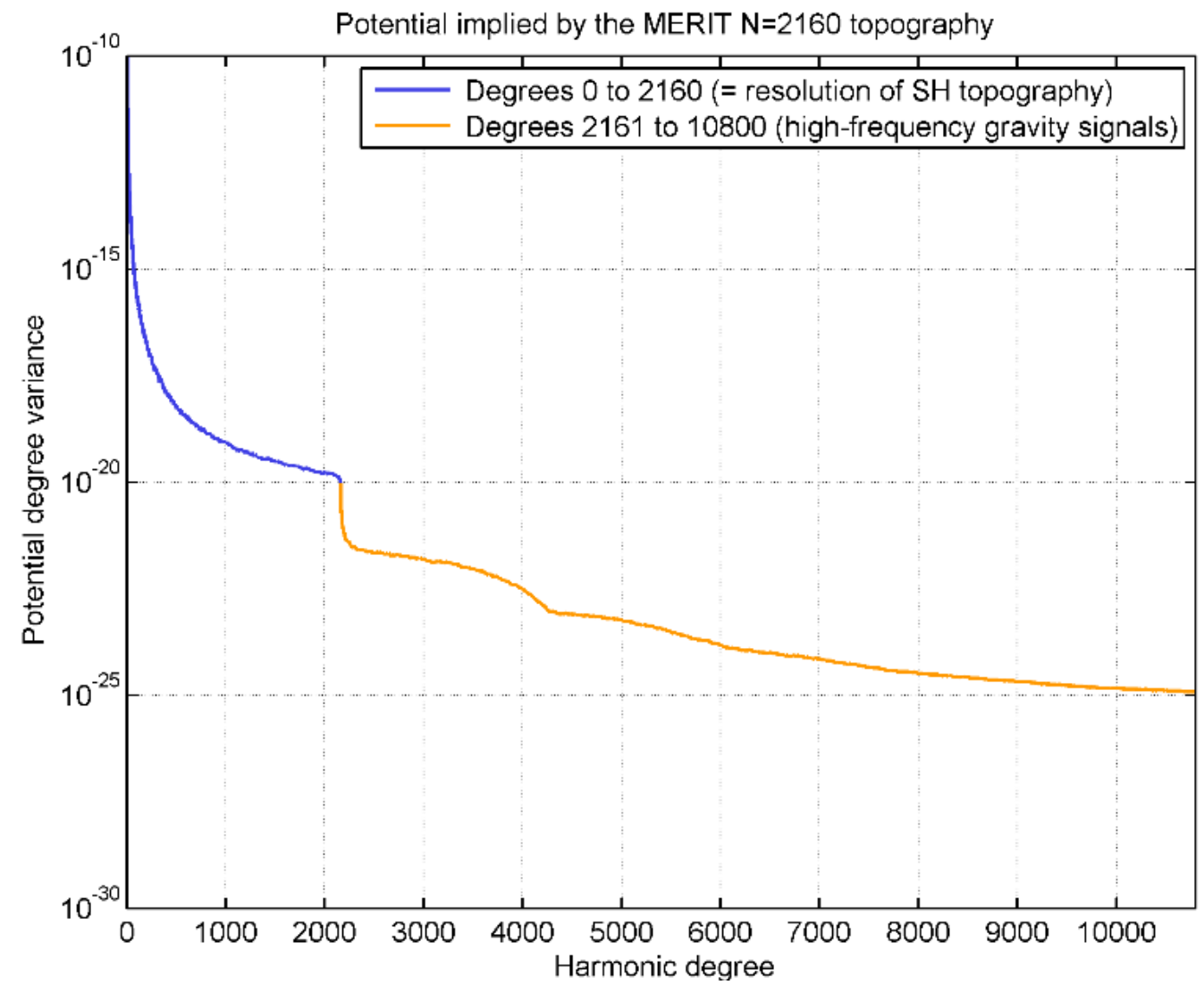

Fig. S1. Dimensionless degree variances of the gravitational potential implied by the MERIT topography. Blue curve: degree variances for the band-width of the input topography $(0 \leq n \leq 2,160)$; orange curve: degree variances associated with the short-scale gravity signals $(2,161 \leq n \leq 10,800)$.

\section{S1.2.2 Spherical harmonic syntheses}

For the spherical harmonic synthesis (SHS) of gravity effects we use the spherical harmonic series (e.g.,Torge and Müller 2012)

$$
\delta g_{N \operatorname{Sin} . . N \max }^{S G M}=\frac{G M}{r^{2}} \sum_{n=N \min }^{N \max }(n+1)\left(\frac{R}{r}\right)^{n} \sum_{m=-n}^{n} \bar{V}_{n m} \bar{Y}_{n m}(\varphi, \lambda)
$$

where the evaluation point $(r=R+H, \varphi, \lambda)$ resides at the surface of the 3 " MERIT topography model, Nmin is the lower harmonic degree and Nmax the upper harmonic degree of evaluation. Given the necessity to synthesize gravity at $\sim 27.9$ billion points with varying topography heights, classical point-by-point evaluation techniques for Eq. (5) would lead to unacceptably large computation times (cf. Hirt 2012). Assuming a computational speed of $1 \mathrm{~s}$ per point for point-wise evaluation of Eq. (5) to ultra-high degree of 10,800, the total computational effort would be on the order of $\sim 5$ million CPU hours.

For a much more efficient evaluation of Eq. (5), so-called 3D-SHS techniques based on gradient continuation are very well suitable, as described in Holmes (2003), Balmino et al. (2012), Hirt (2012) and implemented in the isGrafLab software by Bucha and Janak (2014). The idea behind the 3D-SHS 
technique is to accurately approximate $\delta g_{0 . . N \operatorname{Smax}}^{S G M}$ via a Taylor series that up- or downward-continues gravity effects with $1^{\text {st }}, 2^{\text {nd }}, \ldots q$-th order radial derivatives computed highly efficiently at some constant reference height (e.g., Bucha and Janak 2014). By choosing the order $q$ of Taylor series expansion large enough, approximation errors can be reduced to the microGal-level $\left(1 \mu \mathrm{Gal}=10^{-3}\right.$ $\mathrm{mGal}=10 \mathrm{~nm} \mathrm{~s}^{-2}$ ) or even well below, so become negligible. The adoption of 3D-SHS techniques reduces the computation effort associated with the gravity synthesis for 27.9 billion points to few $10,000 \mathrm{CPU}$ hours (or less than $1 \%$ of the time needed for point-by-point syntheses).

For the SRTM2gravity model generation, we evaluated Eq. (5) in terms of 19,404 $1^{\circ}$ tiles, each containing $1200 \times 1200$ MERIT terrain elevations at 3" spatial resolution. For each tile, its mean elevation is used as constant reference height where gravity values and the radial derivatives are computed. This strategy reduces the radial distances, over which the gravity values are continued, and thus accelerates the convergence of the Taylor series (cf. Hirt 2012, Bucha and Janak 2014).

For the selection of the computational parameters in ultra-high degree 3D-SHS, there are some important aspects to be taken into account:

(1) A band-limited topographic mass model (in our case $0 \leq n \leq 2,160$ ) generates a full-scale gravitational field, with high-frequency signals occurring with associated degrees $n>2,160$ (cf. Hirt and Kuhn 2014), also see Fig. S1. These high-frequency signals can even exceed the $10 \mathrm{mGal}$ level, so must be considered for accurate modelling.

(2) Luckily, in case of a degree-2160 topographic reference model, the bulk of high-frequency signals is contained in the spectral band $2161 \leq n \leq 6,840$ over flat areas and $2161 \leq n \leq$ 10,800 over mountainous areas (e.g., Hirt et al. 2016), such that extremely high-resolution and costly spectral modelling (e.g., to multiples of degree 10,800 ) can be avoided.

(3) Nonetheless there is always the effect of omitted signals because we cannot model the highfrequency signal to infinity. From Hirt et al. (2016, Table 2 ibid), for $\operatorname{Nmax}=10,800$, this effect can be constrained to be globally at the $5 \mu \mathrm{Gal}$ RMS level and maximum error amplitudes will rarely reach $\sim 1-2 \mathrm{mGal}$.

(4) The 3D SHS computation times increase about quadratically with the maximum harmonic degree $N \max$ of evaluation, and linearly with the order $q$ of the Taylor series. The required order $q$ increases with $N \max$ and the roughness of the topography (cf. Hirt 2012, Balmino 2012, Hirt et al. 2016) to keep approximation errors small.

For these reasons, it is necessary to balance computational costs versus the desired quality of the modelling, by choosing Nmax not as high as possible, but instead as low as required to make approximation and omission errors sufficiently small. For efficient ultra-high degree 3D SHS, the topography of all $19,4041^{\circ}$ tiles was classified as mountainous or other (e.g., flat or undulating) terrain. In case of mountainous terrain, the RMS (root-mean-square) of the heights exceeds a value of $1200 \mathrm{~m}$, or the elevation range (that is, maximum minus minimum value) exceeds $5000 \mathrm{~m}$. Thus, the class "mountainous" contains tiles containing extremely steep or rugged mountain topography, as found, e.g., over parts of the Himalayas, Rocky Mountains, but also highly elevated terrain (e.g., parts of Africa or the Andes). Depending on the classification of the individual tiles, Taylor series of varying order $q$ were applied (see Table S1) as trade-off between computation times and approximation errors. Approximation errors associated with the chosen $q$-values are at the RMS level of few $\mu \mathrm{Gal}$, and do not exceed amplitudes of $0.01 \mathrm{mGal}$. This was assessed with higher-order Taylor series expansions with $q=30$ over a test area located in the Himalaya Mountains. The geographic distribution of tiles with classification as flat or rough is shown in Fig. S2. 
Table S1. Parameters $q$ and Nmax used in the synthesis of the long-wavelength gravity signal $n \leq 2160$ and short-scale gravity signal $(n>2160)$ implied by the 2,160 reference topography

\begin{tabular}{|c|c|c|c|c|c|}
\hline \multirow{2}{*}{$\begin{array}{l}\text { Tile classified } \\
\text { as }\end{array}$} & \multirow{2}{*}{$\begin{array}{l}\text { Number of } \\
\# 1^{\circ} \times 1^{\circ} \text { tiles }\end{array}$} & \multicolumn{2}{|c|}{$\begin{array}{l}\text { Long-wavelength gravity signal } \\
\text { of the reference topography }\end{array}$} & \multicolumn{2}{|c|}{$\begin{array}{l}\text { High-frequency gravity signal } \\
\text { of the reference topography }\end{array}$} \\
\hline & & Spectral band & Order $q$ & Spectral band & Order $q$ \\
\hline Mountainous & 2,508 & $0 \leq n \leq 2160$ & 10 & $\begin{array}{c}2161 \leq n \leq \\
N \max =10,800\end{array}$ & 22 \\
\hline $\begin{array}{l}\text { Other (flat, } \\
\text { undulating, ...) }\end{array}$ & 16,804 & $0 \leq n \leq 2160$ & 7 & $\begin{array}{c}2161 \leq n \leq \\
N \max =6,840\end{array}$ & 10 \\
\hline
\end{tabular}

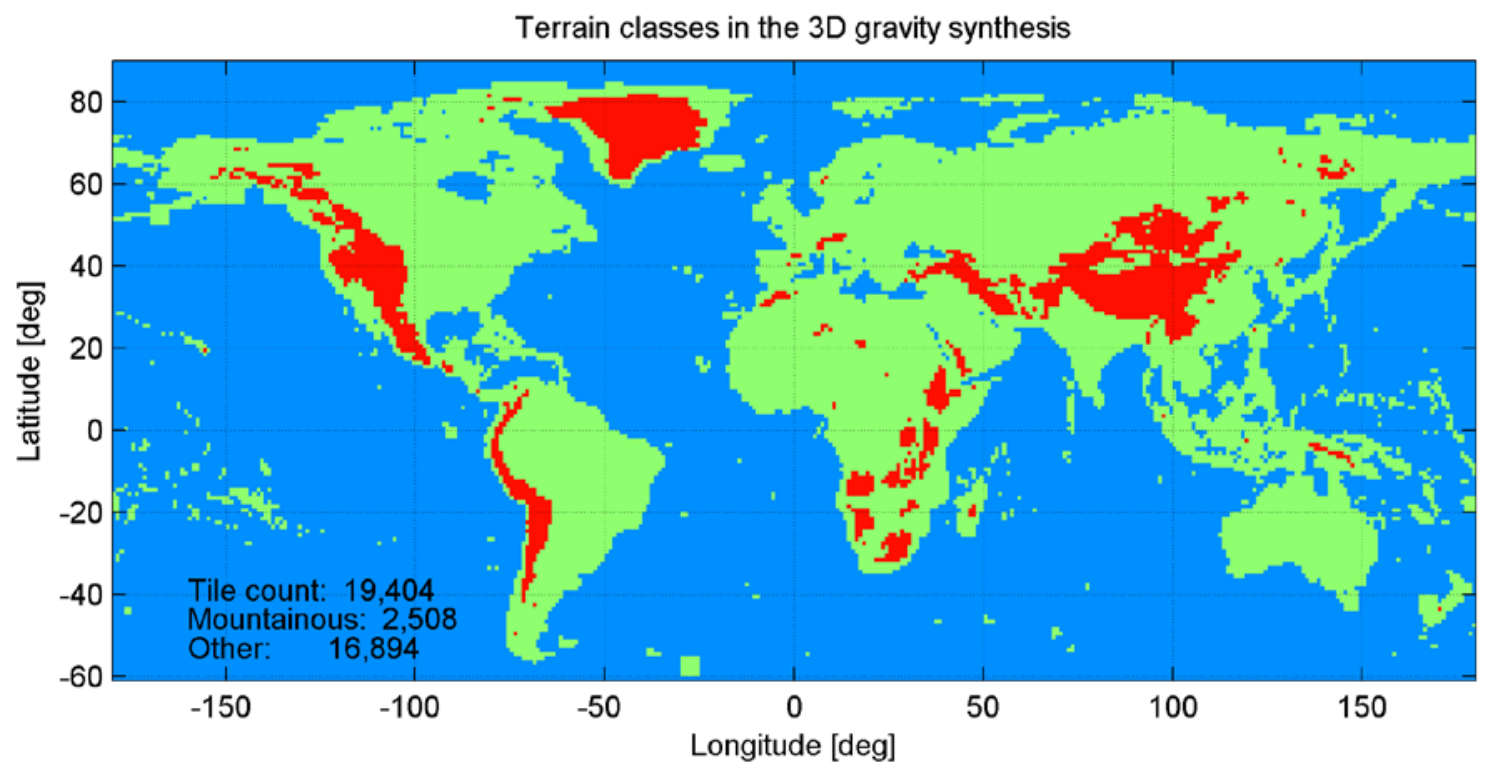

Fig. S2. Geographic distribution of $1^{\circ}$ tiles of the SRTM2gravity model and their terrain type classification for the 3D SHS. Red: classified as mountainous (\#2,508 tiles; Nmax $=10,800$ used in the synthesis); green: other tiles not classified as mountainous (\#16,894; $N \max =6,840$ used in the synthesis). Blue: tiles without synthesized gravity values (the open global ocean areas within $-60^{\circ}$ to $90^{\circ}$ latitude).

With the chosen spectral band-widths for the high-frequency gravity signals ( $2161 \leq n \leq 6,840$ over flat areas and $2161 \leq n \leq 10,800$ over rough areas), the majority of the high-frequency signals generated by the $N=2160$ topographic mass model is very well represented, and the required computation times are kept relatively small. From Hirt et al. (2016), the amplitudes of omitted signals with associated harmonic degrees $n>N \max =6,840$ rarely reach few $0.1 \mathrm{mGal}$ over flat areas, and those over rough topography and $n>\operatorname{Nmax}=10,800$ very rarely exceed $1-2 \mathrm{mGal}$. A further increase in computational effort (e.g., higher values for $\operatorname{Nmax}$ and $q$ ) would not justify the gain in accuracy.

As result of the 3D-SHS computations, we obtained the long-wavelength signal $\delta g_{0 . .2160}^{S G M}$ and shortwavelength signal $\delta g_{2161 . . N \max }^{S G M}$ of the reference topography (Eq. 3), evaluated at the 3" MERIT topographic surface. Its sum

$$
\delta g^{H_{R E F}} \approx \delta g_{0 . .2160}^{S G M}+\delta g_{2161 . . N \max }^{S G M}
$$


closely approximates the gravity signal implied by the reference topographic mass model $\left(H_{R E F}, \rho\right)$ as would be obtained through a global evaluation of Newton's integral in the spatial domain

$$
\delta g^{H_{R E F}}=G \rho \int_{\psi=0}^{\pi} \int_{\alpha=0}^{2 \pi} \int_{r_{Q}=R}^{R+H_{R E F}} \frac{r_{Q}^{2}\left(r-r_{Q} \cos \psi\right)}{l^{3}} d r_{Q} d \alpha \sin \psi d \psi,
$$

see the numerical study by Hirt et al. (2016) for full details. From Hirt et al. (2016, Table 2 ibid), remaining approximation errors - as associated with the choice of Nmax and $q$ - can be constrained to be at the $5 \mu \mathrm{Gal}$ RMS level and maximum error amplitudes will rarely reach $\sim 1-2 \mathrm{mGal}$.

\section{S1.3 Spatial domain residual gravity forward modelling (RTM)}

For the computation of the high-frequency gravity signal $\delta g^{R T M}$ we apply residual terrain modelling (RTM; cf. Forsberg and Tscherning 1981; Forsberg 1984). A residual terrain model, bounded by heights $H$ of the $3^{\prime \prime}$ MERIT topography, and reference heights $H^{R E F}$ (cf. Eq. 3) of the $N=2160 \mathrm{SH}$ expansion of the MERIT topography, together with the mass-density $\rho$, is the topographic mass model used as input. The RTM integral to be evaluated reads in spherical approximation (cf. Hirt et al. 2019)

$$
\begin{aligned}
\delta g^{R T M} & =G \rho \int_{\psi=0}^{\pi} \int_{\alpha=0}^{2 \pi} \int_{r_{Q}=R+H_{R E F}}^{R+H} \frac{r_{Q}^{2}\left(r-r_{Q} \cos \psi\right)}{l^{3}} d r_{Q} d \alpha \sin \psi d \psi \\
& \approx G \rho \int_{\psi=0}^{\psi_{0}} \int_{\alpha=0}^{2 \pi} \int_{r_{Q}=R+H_{R E F}}^{R+H} \frac{r_{Q}^{2}\left(r-r_{Q} \cos \psi\right)}{l^{3}} d r_{Q} d \alpha \sin \psi d \psi
\end{aligned}
$$

where the variables are the same as in Eq. (1). As the decided benefit of the RTM technique for our work, the global integration radius ( $180^{\circ}$ in angular degrees, or $\pi$ in radians) in Eq. (7a) can be replaced by some local integration radius $\psi_{0}$ defining a spherical cap around the computation point (Eq. 7b). By limiting the integration domain to, e.g., $\psi_{0}=0.4^{\circ}$ (Eq. $7 \mathrm{~b}$ ), the number of mass elements to be considered in the numerical integration is significantly reduced, and the computational efficiency increases compared to a global numerical integration (as in Eq. 1). Because topographic heights $H-H_{R E F}$ oscillate between positive and negative values outside the integration radius, their effect on the computed gravity value $\delta g^{R T M}$ largely cancels out (cf. Forsberg 1984).

For the discretisation of the RTM integral (Eq. 7b), TUM in-house software (Yang et al. 2018) is used based on a combination of polyhedra (in the near-zone up to $0.015^{\circ}$ around the computation point), prisms (from $0.015^{\circ}$ to $0.025^{\circ}$ spherical distance), tesseroids (from $0.025^{\circ}$ to $0.06^{\circ}$ ) and point-masses (from $0.06^{\circ}$ to $\psi_{0}=0.4^{\circ}$ ). $3^{\prime \prime}$ MERIT DEM data is used up to a spherical distance of $0.06^{\circ}$, and block average values (30") up to a distance of $\psi_{0}=0.4^{\circ}$. A rotation of the gravitational attraction components from the topocentric coordinate system aligned with the polyhedral/prisms edges to the topocentric coordinate system at the computation point has been performed (cf. Heck and Seitz 2007).

Our specific selection of RTM computation parameters (value $\psi_{0}$, grid resolutions and radii used for switching between different mass-elements) was primarily driven by the necessity to reduce the number of mass elements (and thus the mathematical operations) in the numerical integration such that a target computational speed of $\sim 7$ computation points per second could be achieved. Such a performance is required to complete the SRTM2gravity forward modelling at $\sim 27.9$ billion 
computation points within the available budget class of $\sim 1$ million CPU hours. Approximation errors associated with the chosen integration parameters have been found to be below the RMS level of $\sim 0.1 \mathrm{mGal}$, which is considered here as well acceptable for the quality of the SRTM2gravity modelling.

For computation points located inside the reference topography (that is, when the computation point height $H<H^{R E F}$ ), it is well known that the gravitational potential is non-harmonic (Forsberg and Tscherning 1981) and not suited to describe the external gravitation field. This issue is solved in approximation in SRTM2gravity by applying the so-called harmonic correction (Forsberg and Tscherning 1981)

$$
h c \approx 4 \pi G \rho H_{P}^{R T M}, H_{P}^{R T M}<0
$$

that corresponds to a mass condensation using double Bouguer reduction of planar plate thickness $H_{P}^{R T M}=H-H^{R E F}$. Approximation errors associated with Eq. (8) have been characterized and quantified by Hirt et al. (2019), and corroborated in our validation experiments (Sect. S3) as follows:

- Points with $H \geq H^{R E F}$ (that is, $H_{P}^{R T M} \geq 0$ ) are always unaffected by the harmonic correction approximation errors because Eq. (8) is not applied.

- Approximation errors for points with $H_{P}^{R T M}<0$ were constrained not to exceed a value of $\sim 0.012 \frac{\mathrm{mGal}}{\mathrm{m}} \cdot H^{R T M}$ (Hirt et al. 2019). As a worst case example, over deep valleys of the Himalayas, $H_{P}^{R T M} \approx-1000 \mathrm{~m}$ errors reach amplitudes of $\sim 12 \mathrm{mGal}$ in extreme cases.

The role of harmonic correction approximation errors for the error budget of the SRTM2gravity values is further discussed in Sect. S3.

\section{S2 Supercomputer use}

Given the demanding computational requirements for gravity forward modelling with local resolution and global coverage, the use of some supercomputing platform is inevitable. For the SRTM2gravity project, advanced computational resources were made available through the Leibniz Supercomputing Centre (LRZ) of the Bavarian Academy of Sciences (www.Irz.de). We used the Haswell island of the LRZ's SuperMUC Petascale Computing System. This system consists of a total of 86,016 Central Processing Units (CPUs) arranged in terms of 3,072 nodes. The 28 CPUs of each Haswell node share $64 \mathrm{~GB}$ per node ( $\sim 2.1 \mathrm{~GB}$ available per CPU on average) and were operated at a frequency of $1.8 \mathrm{GHz}$ during our computations.

The SRTM2gravity computational exercise was subdivided into $1^{\circ} \times 1^{\circ}$ geographical tiles, each containing $1200 \times 1200$ computation points at $3^{\prime \prime}$ spatial resolution. Within the MERIT data area from $-60^{\circ}$ to $90^{\circ}$ latitude, there are 19,402 tiles with one or more land points, out of which 14,476 extend over the SRTM data area from $-58^{\circ}$ to $+60^{\circ}$ latitude, and another 4,926 tiles are located in Arctic regions ( $+60^{\circ}$ to $85^{\circ}$ latitude) For each tile, three computation runs were carried out:

- SH synthesis of gravity effects implied by the degree-2,160 MERIT topography at the 3" MERIT topographic surface in band $0 \leq n \leq 2,160$

- $\quad$ SH synthesis of high-frequency (HF) gravity effects implied by the degree-2,160 MERIT topography at the 3" MERIT topographic surface

- in band $2161 \leq n \leq 10,800$ (over mountainous topography) or

- in band $2161 \leq n \leq 6,480$ (elsewhere, cf. Fig. S2 for the classification).

- Forward modelling of high-frequency gravity effects in the spatial domain using the RTM technique and $0.4^{\circ}$ integration caps around each computation point. 
Table $\mathbf{S} 2$ reports the run-times per computation run type and tile, and resulting computation times.

Table S2. Number of tiles, measured computation times for the three kinds of computations (SGM = synthesis in band 0 to $2160, \mathrm{HF}=$ synthesis in band 2161-6840/10800 and RTM = residual terrain modelling).

\begin{tabular}{lrrrrrrrr}
\hline Terrain type & \multicolumn{4}{c}{$\begin{array}{c}\text { Measured run } \\
\text { times per tile }(\mathbf{h})\end{array}$} & \multicolumn{3}{c}{ Required CPU hours (h) } \\
\hline Terrain type & \# Tiles & SGM & HF & RTM & SGM & HF & \multicolumn{1}{c}{ RTM } & \multicolumn{1}{l}{ Total } \\
\hline Mountainous & 2,508 & 0.4 & 5.5 & 57.5 & 1,045 & 11,286 & 142,956 & 155,287 \\
Other & 16,894 & 0.4 & 1.5 & 57.5 & 7,039 & 25,341 & 962,958 & 995,338 \\
Total & 19,402 & & & & 8,804 & 36,627 & $1,105,914$ & $\mathbf{1 , 1 5 0 , 6 2 5}$ \\
\hline
\end{tabular}

\section{S3 Validation experiments}

For validation of the SRTM2gravity modelling technique described in Sect. S1, we have evaluated Newton's integral (Eq. 1) over selected test areas through a full-scale global numerical integration in the spatial domain. These experiments use the same 3" MERIT topographic mass model, and identical numerical values for constants $\rho, R$ and $G$, but do not involve any spectral-domain calculations, do not use SH reference surfaces (Sect. S1.2), nor residual terrain modelling (Sect. S.1.3). As such, the global numerical integration provides a check on the SRTM2gravity calculations with fully independent methodology.

We have defined six $2^{\circ} \times 2^{\circ}$ test areas around the globe as follows (cf. Table S3 and Fig. S3)

- Himalyas (including the Southern flanks and the Mount Everest summit, as example of extremely rugged topography with an elevation range of $\sim 8000 \mathrm{~m}$ ),

- Switzerland (as example for an Alpine region with an elevation range of $\sim 4000 \mathrm{~m}$ and shortscale terrain variations of $\pm 2000 \mathrm{~m}$ ),

- Canada (covering parts of the Rocky Mountains, including high-latitudes up to $60^{\circ}$ and landsea transitions, elevation range $\sim 4500 \mathrm{~m}$ ),

- South America (covering parts of the Andes Plateau with an elevation range of $\sim 5000 \mathrm{~m}$ )

- Indonesia (covering several land-sea transitions; straddles the equator to test the software, elevation range $\sim 2500 \mathrm{~m}$ ),

- Australian Alps (most elevated part of Australia, but on a global scale rather smooth terrain, second area on the Southern Hemisphere, elevation range $2000 \mathrm{~m}$ ).

The test areas generally focus on mountainous or extremely mountainous terrain where forward modelling errors can be expected to be the largest. Further, they cover landmass of the Northern and Southern, Eastern and Western hemispheres to provide spot-checks on SRTM2gravity around the globe.

To evaluate Newton's integral (Eq. 1) in the spatial domain, Curtin's in-house software (e.g. Kuhn and Hirt 2016) has been used based on tesseroids approximated by mass-equal prisms (up to $6^{\circ}$ around the computation point) and tesseroids to cover the whole globe $\left(\psi_{0}=\pi\right)$. The software performs a truly-global numerical integration of mass-density effects. It has been tested to provide topographic gravity at a precision level well below 0.1 mGal (e.g., Hirt and Kuhn 2014, Kuhn and Hirt 2016, Hirt et al. 2016) thus being suitable to be used for validation. In order to reduce computation time, cascading grid resolutions have been used with $3^{\prime \prime}$ in the near vicinity around the computation point (up to an extension of 20') and coarser resolutions farther away, e.g. 15', $1^{\prime}, 3^{\prime}$ and $15^{\prime}$ with respective radii of $2^{\circ}, 6^{\circ}, 15^{\circ}$ and global. The cascades have been empirically selected so that approximation errors remain at most at the $\mu \mathrm{Gal}$ level. Based on this configuration and using advanced computational resources provided by Pawsey's Supercomputing Centre 
(www.pawsey.org.au) a computation speed of $\sim 2.5$ s per point could be achieved requiring $\sim 24,000$ CPU hours to provide the reference solutions over the six selected validation areas.

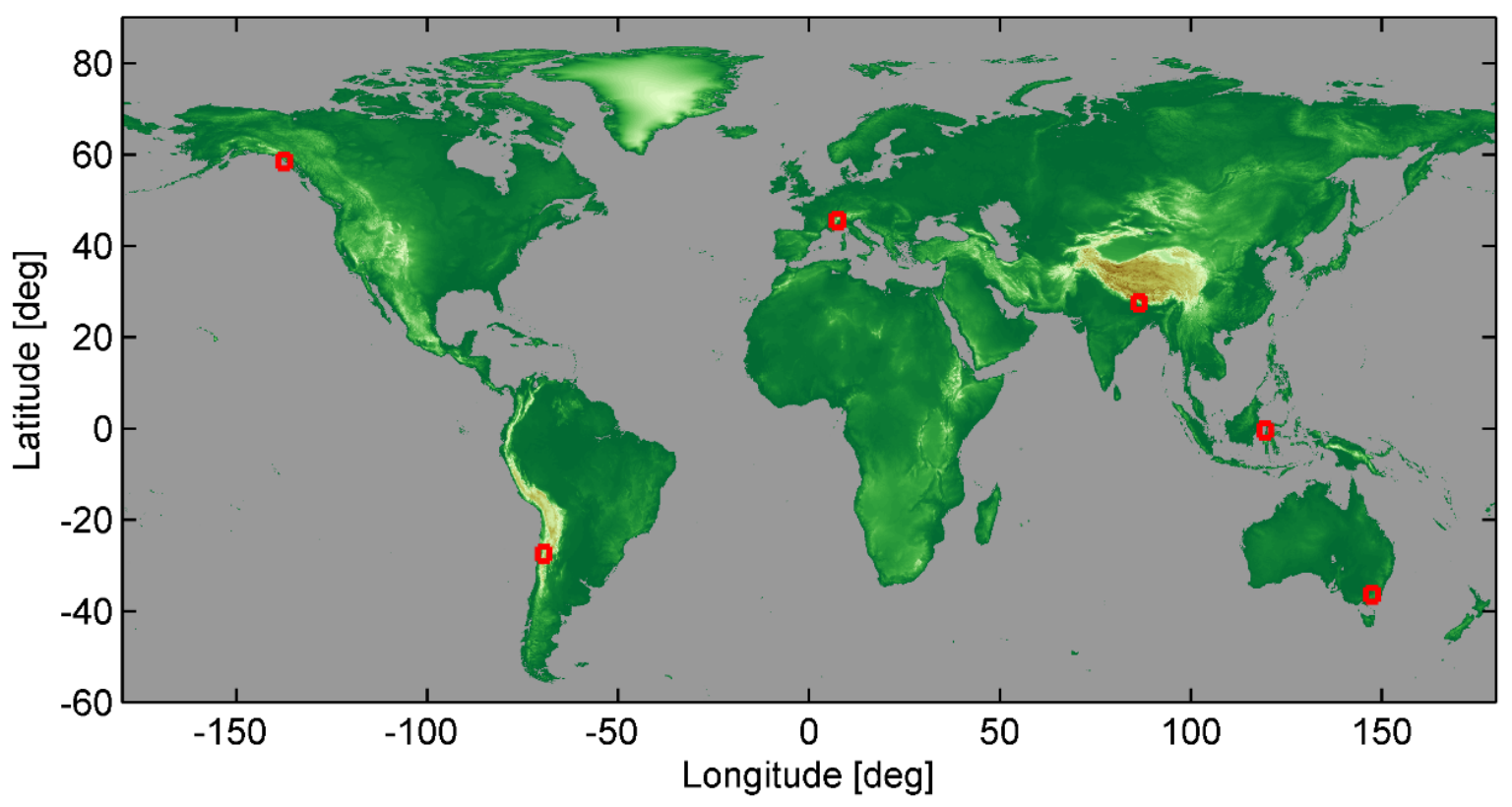

Fig.S3. Location of the six test areas around the globe

Tab. S3. Test areas and geographic boundaries in degrees. Each area contains 5,760,000 $(=2,400 \times 2,400)$ computation points at 3 " resolution in cell-centred grid node registration.

\begin{tabular}{lcccc}
\hline Area name & $\begin{array}{c}\text { Minimum } \\
\text { Longitude }\end{array}$ & $\begin{array}{c}\text { Maximum } \\
\text { Longitude }\end{array}$ & $\begin{array}{c}\text { Minimum } \\
\text { Latitude }\end{array}$ & $\begin{array}{c}\text { Maximum } \\
\text { Latitude }\end{array}$ \\
\hline Himalayas & 86 & 88 & 27 & 29 \\
Switzerland (European Alps) & 7 & 9 & 45 & 47 \\
Canada (Rocky Mountains) & -138 & -136 & 58 & 60 \\
South America (Andes) & -70 & -68 & -28 & -26 \\
Indonesia (Islands) & 119 & 121 & -1 & 1 \\
Australia (Australian Alps) & 147 & 149 & -37 & -35 \\
\hline
\end{tabular}

Tab. S4. Descriptive statistics of the differences between gravimetric terrain corrections from full-scale numerical integration and SRTM2gravity, as released to the public. Over each $2^{\circ} \times 2^{\circ}$ area, 5,760,000 values were compared. All values in $\mathrm{mGal}$

\begin{tabular}{lcccc}
\hline Area name & Minimum & Maximum & Mean & RMS \\
\hline Himalayas & -6.60 & 12.06 & 0.02 & 0.75 \\
Switzerland (European Alps) & -9.60 & 7.34 & -0.09 & 0.57 \\
Canada (Rocky Mountains) & -5.05 & 9.12 & -0.08 & 0.46 \\
South America (Andes) & -4.75 & 8.12 & -0.03 & 0.17 \\
Indonesia (Islands) & -3.18 & 3.34 & -0.02 & 0.14 \\
Australia (Australian Alps) & -6.53 & 11.41 & -0.05 & 0.11 \\
\hline
\end{tabular}



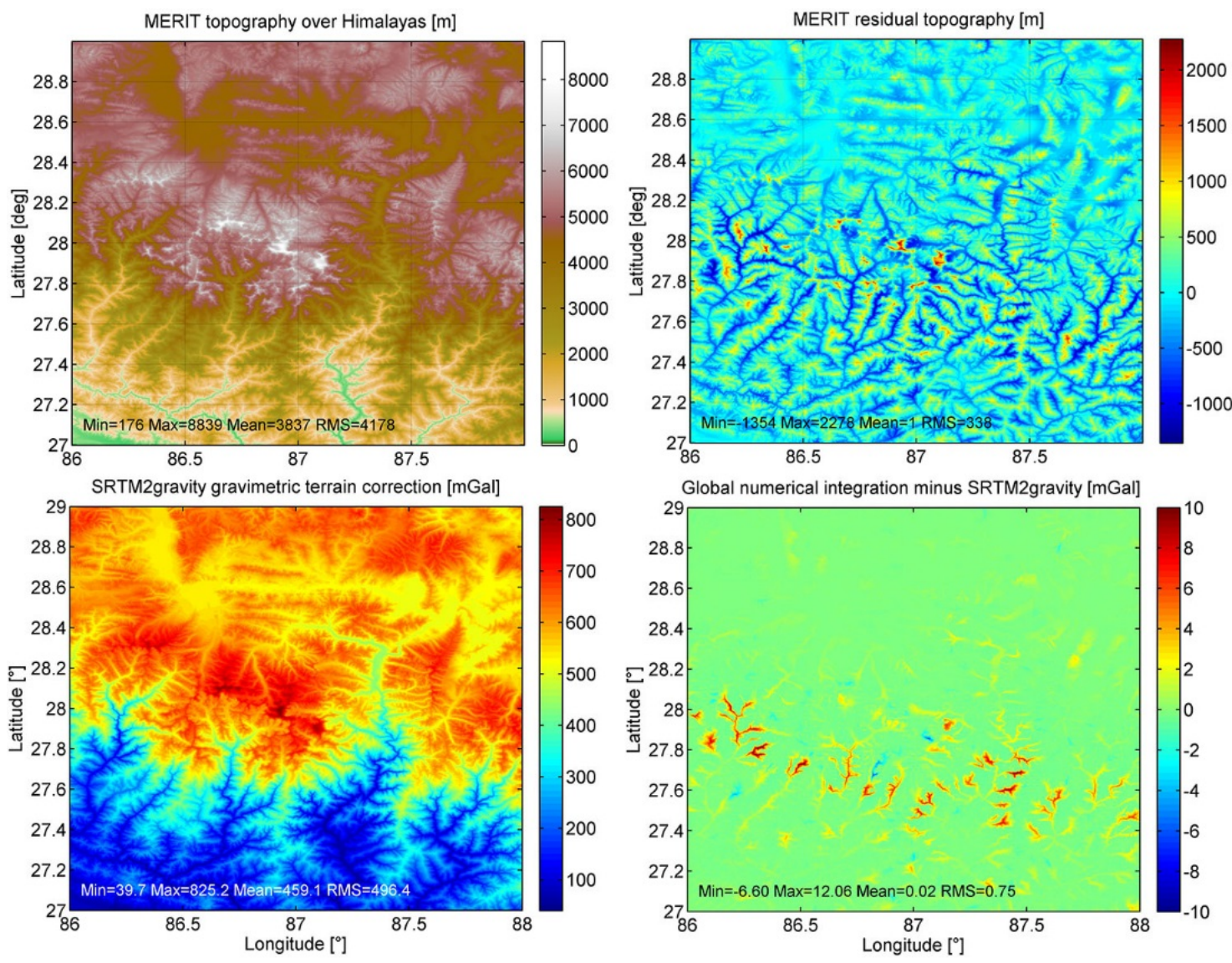

Fig. S4. Results of the validation experiment over the Himalaya test area
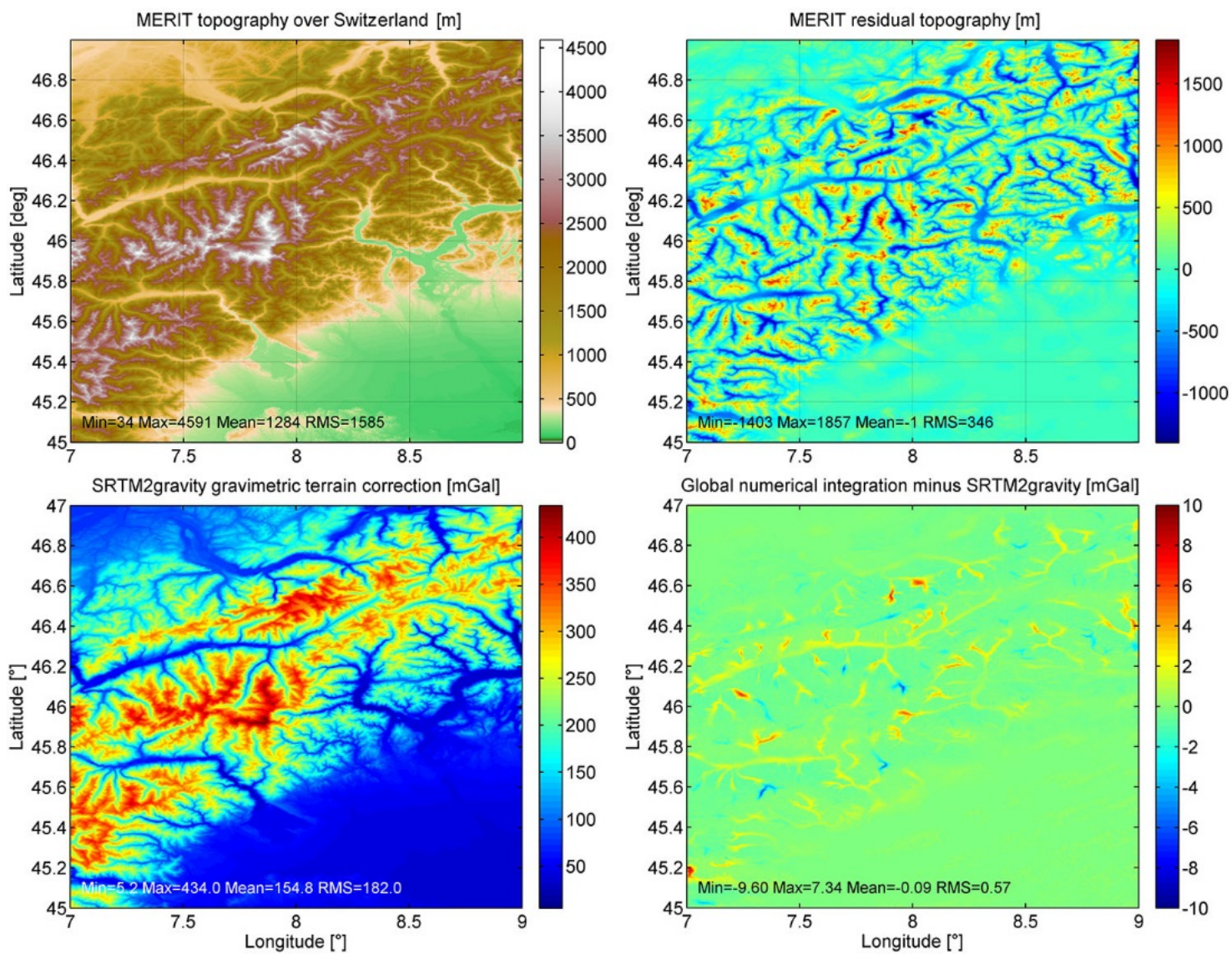

Fig. S5. Results of the validation experiment over the test area Switzerland 

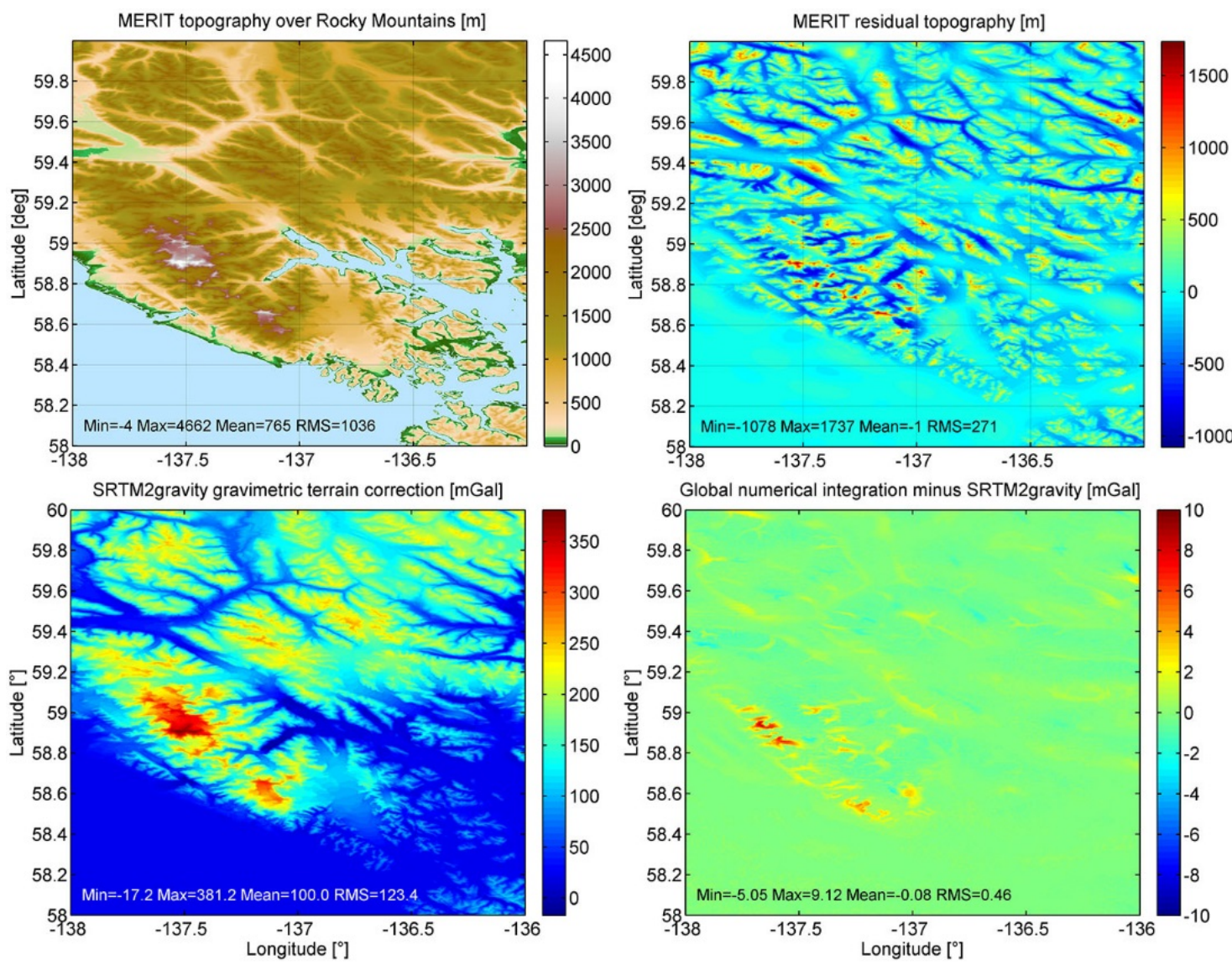

Fig. S6. Results of the validation experiment over the test area Rocky Mountains
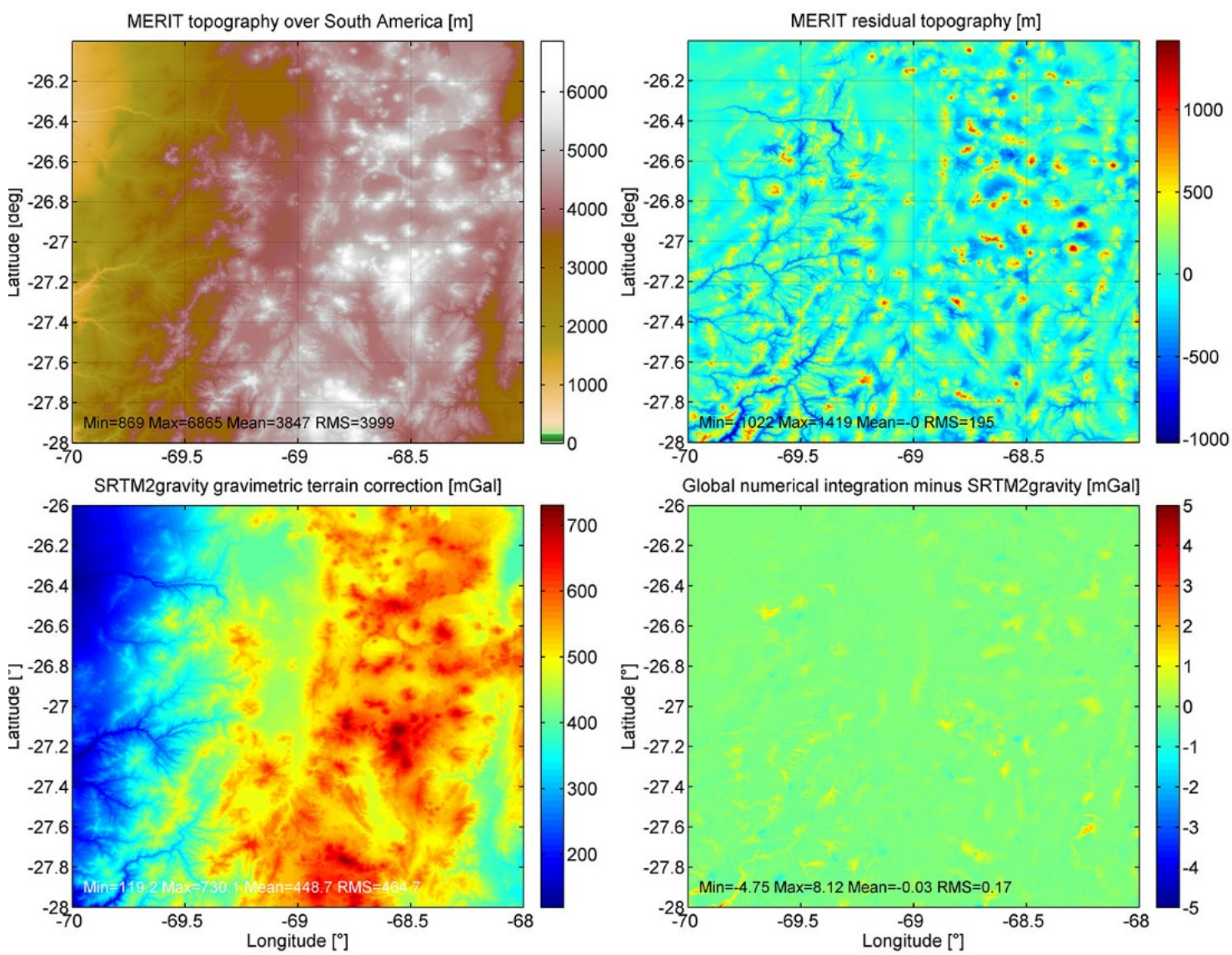

Fig. S7. Results of the validation experiment over the test area Andes Mountains 

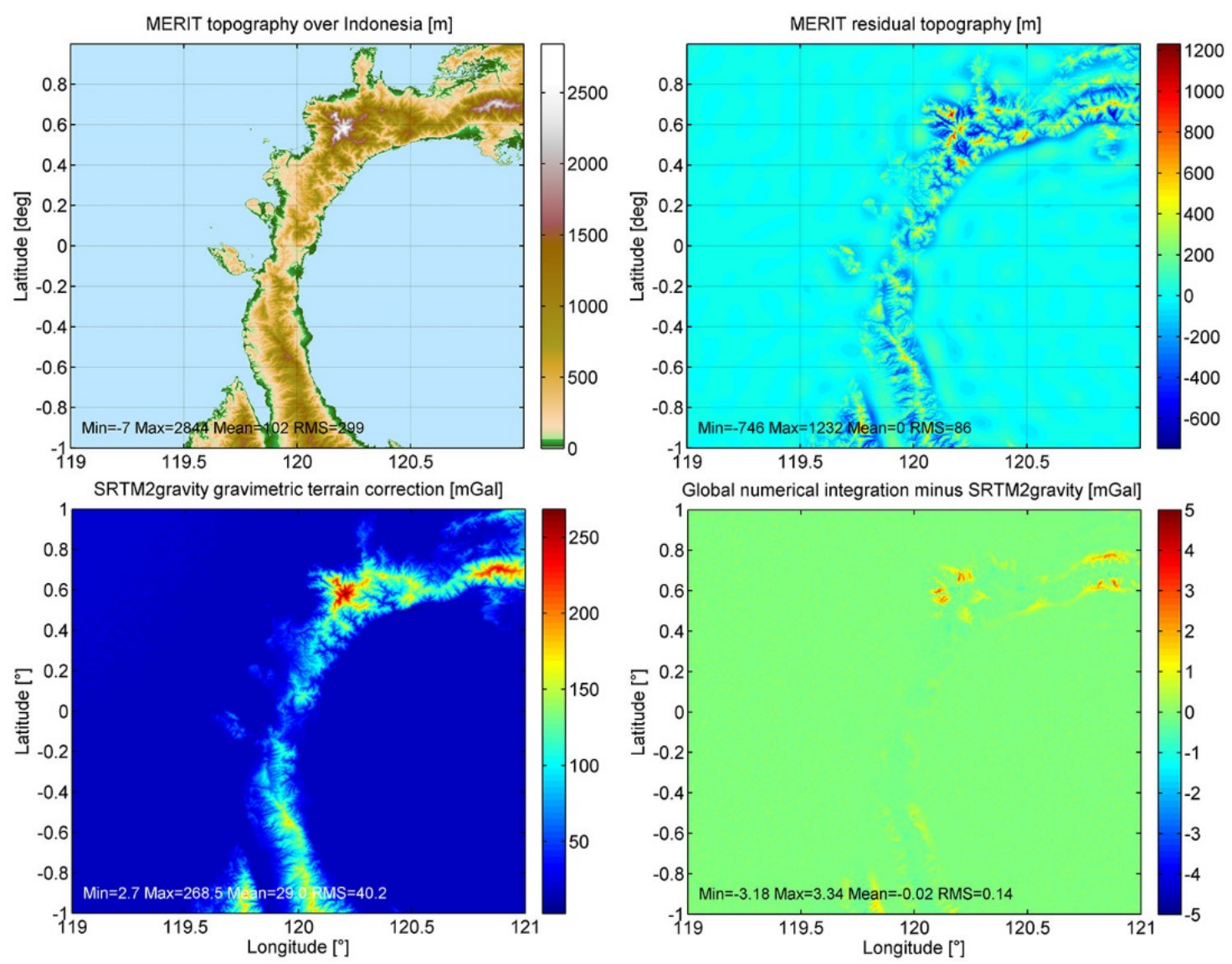

Fig. S8. Results of the validation experiment over the test area Indonesia
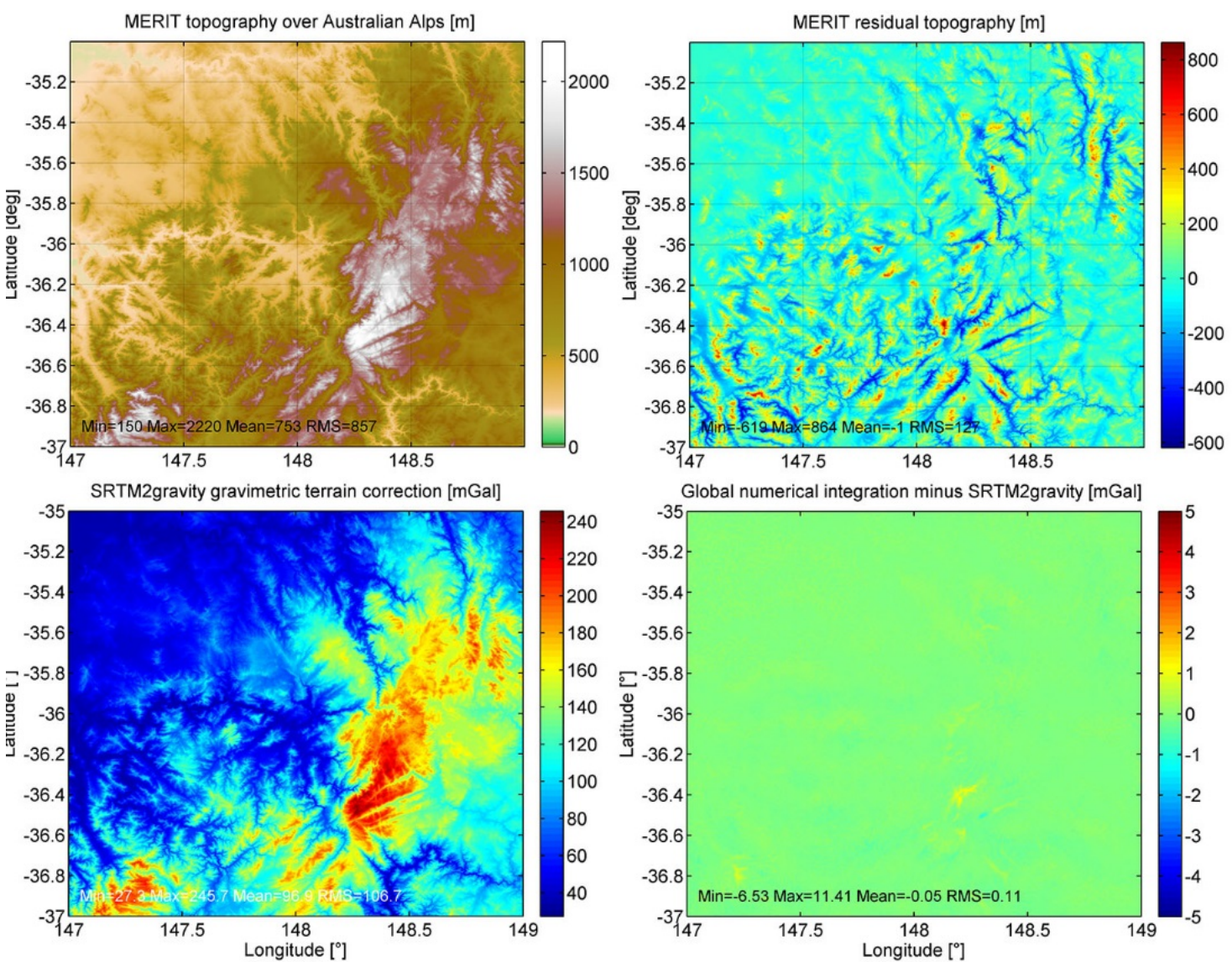

Fig. S9. Results of the validation experiment over the test area Australian Alps 
For the six test areas, Figs. S4 to S9 show data and results of the validation experiments. Each figure shows the MERIT topography in metres (top left), the residual MERIT topography to exemplify the terrain variations in the RTM forward modelling (top right), the SRTM2gravity gravimetric terrain correction in $\mathrm{mGal}$ (bottom left) and the differences between the full-scale global numerical integration (not shown) and SRTM2gravity gravimetric terrain corrections in mGal (bottom right).

From Figs. S4 to S9 and Table S4, the root-mean-square (RMS) agreement between SRTM2gravity and the reference solution is always better than the $1 \mathrm{mGal}$ level over any of the six test areas. The RMS values are largest for the Himalaya area $(0.75 \mathrm{mGal})$, followed by the European Alps and Canada (0.57 and $0.46 \mathrm{mGal})$. Over the Andes and Indonesian Islands, the RMS agreement is better than 0.2 $\mathrm{mGal}$, and over the Australian Alps as a moderately rugged area, the validation experiment shows an excellent RMS agreement at the level of $\sim 0.1 \mathrm{mGal}$.

Given that more than $90 \%$ of the $19,4041^{\circ} \times 1^{\circ}$ tiles of the SRTM2gravity model feature smoother topography than that of the Australian Alps (using the elevation range across the tile as criterion), we consider the 0.1- 0.2 mGal level representative for wide parts of Earth's land areas, as shown in green in Fig. S10. Opposed to this, larger RMS errors at the $0.5 \mathrm{mGal}$ level are to be expected for Alpine terrain (elevation variation between 2.2 and $5 \mathrm{~km}$ ) and $\sim 0.7-0.8 \mathrm{mGal}$ over extremely rugged terrain (elevation variation larger than $5 \mathrm{~km}$ ). Note that the classification of terrain according to elevation range was made in an attempt to generalize the results of the six validation experiments to the SRTM2gravity data area.

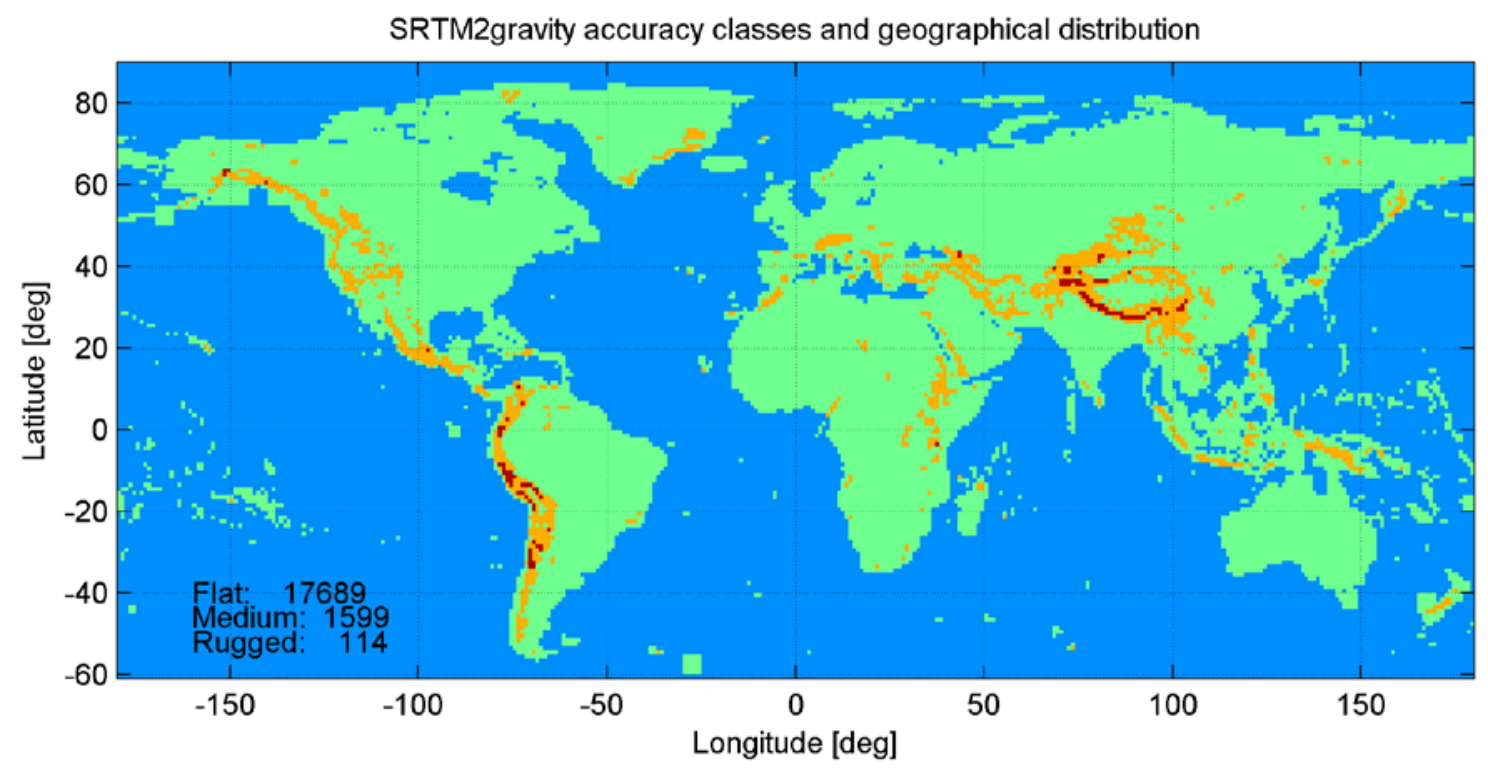

Fig. S10. Accuracy classes assigned to all $1^{\circ} \times 1^{\circ}$ tiles based on the elevation range across the tile and results from the validation experiments. Green: accuracy of $\sim 0.1$ to $\sim 0.2 \mathrm{mGal}$ (for tiles with terrain similar or smoother than that of the Australian Alps), orange: accuracy of $\sim 0.5$ to $\sim 0.6 \mathrm{mGal}$ (for tiles with terrain similar to that of the European Alps, dark red: accuracy of $\sim 0.7$ to $\sim 0.8 \mathrm{mGal}$ (for tiles with elevation variability larger than $5 \mathrm{~km}$ ).

Note that errors at individual SRTM2gravity computation points are largest where residual terrain heights (cf. Figs S4-S9, top right) are strongly negative. This is related to the approximative character of the harmonic correction that was applied in the RTM forward modelling at all computation points located inside the long-wavelength reference topography. These approximation errors tend to increase with the negative RTM elevation, i.e., can be largest in deep and narrow mountain valleys. From Hirt et al. (2019), maximum amplitudes of approximation errors associated the harmonic correction were constrained to $\sim 0.012 \mathrm{mGal} / \mathrm{m}$. For instance, with $-1000 \mathrm{~m}$ RTM elevation, the 
amplitude of the approximation error can reach $\sim 12 \mathrm{mGal}$ in the worst case, but are mostly smaller (see Figs. S4-S9 and Table S2). Over the entire model data area (Fig. 1 in the manuscript), there are less than $0.005 \%$ of computation points with RTM elevations of smaller than $-1000 \mathrm{~m}$, and less than $1 \%$ of computation points with RTM elevations of smaller than $-300 \mathrm{~m}$ (cf. Fig. S11). Therefore, the approximation errors associated with the harmonic correction play a minor role for the overall model quality. We note that points with positive RTM elevation (of arbitrary amplitude) are unaffected by this kind of approximation error, and residuals are always at the RMS-level of 0.1-0.2 mGal. While it is important to be aware of the somewhat reduced quality of SRTM2gravity over deep and narrow valleys, an error level of 0.1-0.2 mGal can be expected over $95 \%$ Earth's land areas. The overall accuracy target for the SRTM2gravity terrain corrections of $\sim 1 \mathrm{mGal}$ has been fully reached or exceeded.

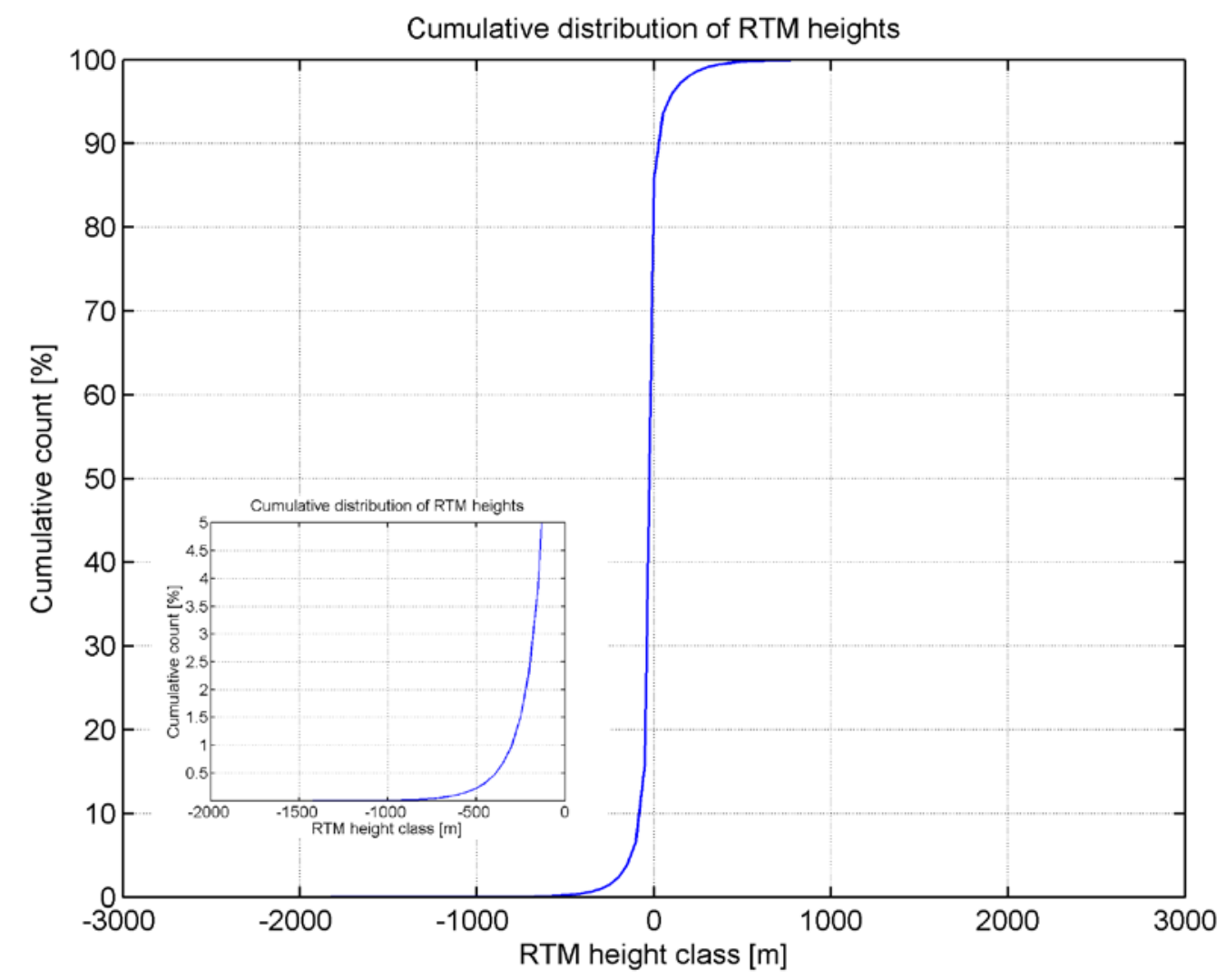

Fig. S11. Cumulative distribution of 27,938,880,000 RTM heights within the SRTM2gravity data area.

\section{S4 Comparison with ground-truth data}

For additional validation of the SRTM2gravity model, we also use ground-truth gravity data sets from terrestrial gravimetry over four countries or regions: Switzerland, Bavaria, Slovakia and Australia. The specifications of the four data sets are summarized in Table S4. While the validation of the full-scale SRTM2gravity product is rather difficult with ground-truth data (the differences show the Bouguer gravity signal, also see Fig. 2 in the manuscript), the residual (short-scale or RTM) gravity component of the SRTM2gravity model can be used to augment a global geopotential model (GGM) such as EGM2008 at short spatial scales. A similar principle was already applied in the development of GGMplus (Hirt et al. 2013), where gravity from a degree-2190 spherical harmonic GGM, based on GRACE and GOCE and EGM2008 data, was augmented with short-scale gravity information from forward modelling (released as the ERTM2160 model, cf. Hirt et al. 2014) and compared with groundtruth data sets. The following procedure to compare ground-truth gravity data with modelled gravity values was followed: 
(1) Calculation of gravity implied by the GRS80 normal gravity field at the 3D location of the gravity station (as defined through geodetic latitude, longitude and the ellipsoidal height).

(2) Subtraction of the normal gravity (1) from the gravity observation, the result are gravity disturbances (radial derivatives of the disturbing potential)

(3) Comparison of the "observed" gravity disturbances from (2) with modelled values obtained by bi-cubic interpolation at the gravity station using the following models
(a) GGMplus,
(b) GGMplus minus ERTM2160 plus residual SRTM2gravity.

In the second case, the short-scale forward-modelled gravity value of GGMplus is effectively replaced with the SRTM2gravity residual component. For each of the ground-truth data sets, Table S5 reports the descriptive statistics of the comparison between observed and modelled gravity disturbances. The RMS (root-mean-square) agreement is found to be at the level of $\sim 2 \mathrm{mGal}$ for the Bavarian data set, $\sim 4 \mathrm{mGal}$ for the Swiss data set and $\sim 3 \mathrm{mGal}$ for the Australian and Slovakian gravity data. Comparing the two model variants (a) and (b), the agreement is comparable or slightly better when the SRTM2gravity model is used. This result is within the expectation, given the (i) higher spatial resolution of the SRTM2gravity modelling ( 3 " vs. 7.2" in ERTM2160), (ii), the use of bare-ground elevations in SRTM2gravity and the consideration of the high-frequency RTM correction in SRTM2gravity (Table S1).

Table S4. Summary of ground-truth data sets used for comparisons with SRTM2gravity

\begin{tabular}{llcll}
\hline Country or region & \multicolumn{2}{l}{$\begin{array}{l}\text { Boundary of data area } \\
\text { Latitude }\end{array}$} & $\begin{array}{l}\text { Number of } \\
\text { stations }\end{array}$ & Data provider or data set reference \\
\hline Bavaria & $47^{\circ}-51^{\circ}$ & $9^{\circ}-14^{\circ}$ & 17,586 & $\begin{array}{l}\text { Landesamt für Digitalisierung, } \\
\text { Breitband und Vermessung (LDBV), } \\
\text { Dipl.-Ing. Franz Lindenthal }\end{array}$ \\
$\begin{array}{l}\text { Switzerland } \\
\text { Australia }\end{array}$ & $45.5-48^{\circ}$ & $5.5^{\circ}-11^{\circ}$ & 31,598 & $\begin{array}{l}\text { Swisstopo, Dr Urs Marti } \\
\text { Geoscience Australia, National } \\
\text { Gravity Data Base (NGDB) }\end{array}$ \\
Slovakia & $-45^{\circ}--^{\circ}$ & $115^{\circ}-155^{\circ}$ & $1,624,972$ & Zahorec et al. (2017) \\
\hline
\end{tabular}

Table S5. Descriptive statistics of the differences between ground-truth gravity disturbances and modelled gravity disturbances. Unit in mGal.

\begin{tabular}{lcccccccc}
\hline Area name & \multicolumn{3}{c}{ (a) Ground-truth minus GGMplus } & \multicolumn{3}{c}{ (b) Ground-truth minus (GGMplus - } \\
ERTM2160 + residual SRTM2gravity)
\end{tabular}

\section{References}

Balmino, G. (1994), Gravitational potential harmonics from the shape of a homogenous body. Celestial Mechanics and Dynamic Astronomy, 60, 331-364.

Balmino, G., N. Vales, S. Bonvalot and A. Briais (2012), Spherical harmonic modelling to ultra-high degree of Bouguer and isostatic anomalies, J. Geod., 86(7), 499-520, doi: 10.1007/s00190-011-0533-4.

Bucha, B. and J. Janák (2014), A MATLAB-based graphical user interface program for computing functionals of the geopotential up to ultra-high degrees and orders: Efficient computation at irregular surfaces, Comp. and Geosci., 66, 219227, doi: 10.1016/j.cageo.2014.02.005. 
Chao, B.F. and D.P. Rubincam (1989), The gravitational field of Phobos. Geophysical Research Letters, 16(8), 859-862.

Forsberg R. and C.C. Tscherning (1981), The use of height data in gravity field approximation by collocation, J. Geophys. Res., 86, 7843-7854.

Forsberg R. (1984), A study of terrain reductions, density anomalies and geophysical inversion methods in gravity field modelling. Report 355, Department of Geodetic Science and Surveying, Ohio State University, Columbus.

Fowler, C.R.M. (2005), The Solid Earth - an introduction to global geophysics, 2nd edition, Cambridge: Cambridge University Press.

Freeden W., and F. Schneider (1998), Wavelet approximations on closed surfaces and their application to boundary-value problems of potential theory. Mathematical Methods in the Applied Sciences, 21,129-163.

Heck, B., and K. Seitz (2007), A comparison of the tesseroid, prism and point-mass approaches for mass reductions in gravity field modelling. J Geod., 81(2), 121-136, doi: 10.1007/s00190-006-0094-0.

Hirt, C. (2012), Efficient and accurate high-degree spherical harmonic synthesis of gravity field functionals at the Earth's surface using the gradient approach, J. Geod., 86(9), 729-744, doi:10.1007/s00190-012-0550-y.

Hirt, C., S.J. Claessens, T. Fecher, M. Kuhn, R. Pail, M. Rexer (2013), New ultra-high resolution picture of Earth's gravity field, Geophysical Research Letters, Vol 40, doi: 10.1002/grl.50838.

Hirt, C., and M. Kuhn (2014), Band-limited topographic mass distribution generates full-spectrum gravity field: Gravity forward modeling in the spectral and spatial domains revisited, J. Geophys. Res., 119(4), 3646-3661, doi: 10.1002/2013JB010900.

Hirt C., M. Kuhn, S.J. Claessens, R. Pail, K. Seitz, T. Gruber (2014), Study of the Earth's short-scale gravity field using the ERTM2160 gravity model, Computers \& Geosciences, 73, 71-80. doi: 10.1016/j.cageo.2014.09.00.

Hirt, C., E. Reußner, M. Rexer, and M. Kuhn (2016), Topographic gravity modelling for global Bouguer maps to degree 2,160: Validation of spectral and spatial domain forward modelling techniques at the 10 microgal level, J. Geophys. Res., 121(9), 6846-6862, doi: 10.1002/2016JB013249.

Hirt, C., B. Bucha ., M. Yang, and M. Kuhn (2019), A numerical study of residual terrain modelling (RTM) techniques and the harmonic correction using ultra-high degree spectral gravity modelling, post-revision, J. Geod.

Holmes S.A. (2003), High degree spherical harmonic synthesis for simulated earth gravity modelling. PhD Thesis, Department of Spatial Sciences, Curtin University of Technology, Perth, Australia.

Jacoby, W., and P.L. Smilde (2009), Gravity interpretation: Fundamentals and application of gravity inversion and geological interpretation, Springer, Berlin, Heidelberg.

Kuhn, M. and C. Hirt (2016), Topographic gravitational potential up to second-order derivatives: an examination of approximation errors caused by rock-equivalent topography (RET), Journal of Geodesy, 90(9), 883-902, doi:10.1007/s00190016-0917-6.

Rexer, M. and C. Hirt (2015), Ultra-high degree surface spherical harmonic analysis using the Gauss- Legendre and the Driscoll/Healy quadrature theorem and application to planetary topography models of Earth, Moon and Mars, Surveys in Geophysics, 36(6), 803-830, doi: 10.1007/s10712-015-9345-z.

Rummel, R., R. H. Rapp, H. Sünkel, and C. C. Tscherning. (1988), Comparisons of global topographic/isostatic models to the Earth's observed gravity field. Report No 388, Dep. Geodetic Sci. Surv., Ohio State Univ., Columbus, Ohio.

Torge W., and J. Müller (2012), Geodesy. 4th Edition. Berlin, New York: W. de Gruyter.

Yamazaki, D., D. Ikeshima, R. Tawatari, T. Yamaguchi, F. O'Loughlin, J.C. Neal, C.C. Sampson, S. Kanae, P.D. Bates (2017), A high accuracy map of global terrain elevations, Geophysical Research Letters, 44(11), 5844-5853, Doi: 10.1002/2017GL072874.

Yang, M., C. Hirt, R. Pail and R. Tenzer (2018), Experiences with the use of mass density maps in residual gravity forward modelling, Studia Geophysica et Geodaetica, 62 (2018), 596-623, doi: 10.1007/s11200-017-0656-z.

Zahorec P., Pašteka R., Mikuška J., Szalaiová V., Papco J., Kušnirák D., Pánisová J., Krajnák M., Vajda P., Bielik M., Marušiak I. (2017), National Gravimetric Database of the Slovak Republic. In: Pašteka R, Mikuška J, Meurers B (eds) Understanding the Bouguer Anomaly, Elsevier, pp 113-125, doi: 10.1016/B978-0-12-812913-5.00006-3. 